MATHEMATICS OF COMPUTATION

Volume 66, Number 217, January 1997, Pages 45-67

S 0025-5718(97)00771-0

\title{
A QUASI-OPTIMAL ERROR ESTIMATE FOR A DISCRETE SINGULARLY PERTURBED APPROXIMATION TO THE PRESCRIBED CURVATURE PROBLEM
}

\author{
MAURIZIO PAOLINI
}

\begin{abstract}
Solutions of the so-called prescribed curvature problem $\min _{A \subseteq \Omega} \mathcal{P}_{\Omega}(A)-\int_{A} g(x), g$ being the curvature field, are approximated via a singularly perturbed elliptic PDE of bistable type. For nondegenerate relative minimizers $A \subset \subset \Omega$ we prove an $\mathcal{O}\left(\epsilon^{2}|\log \epsilon|^{2}\right.$ ) error estimate (where $\epsilon$ stands for the perturbation parameter), and show that this estimate is quasi-optimal. The proof is based on the construction of accurate barriers suggested by formal asymptotics. This analysis is next extended to a finite element discretization of the PDE to prove the same error estimate for discrete minima.
\end{abstract}

\section{INTRODUCTION}

Given an open bounded domain $\Omega$ in $\mathbf{R}^{n}$ with Lipschitz continuous boundary and a curvature field $g \in L^{\infty}(\Omega)$, we address the solution of the so-called prescribed curvature problem, which consists of minimizing the functional

$$
\tilde{\mathcal{G}}(A)=\mathcal{P}_{\Omega}(A)-\int_{A} g(x) d x, \quad A \subseteq \Omega .
$$

Here, $\mathcal{P}_{\Omega}(A)$ denotes the perimeter in $\Omega$ of the Caccioppoli set $A \subseteq \Omega$, i.e., the $(n-1)$-dimensional Hausdorff measure of $\partial^{*} A \cap \Omega$, where $\partial^{*} A$ is the reduced boundary and coincides with $\partial A$ for regular sets [17]. Problems involving a surface energy together with a volume term arise in many fields, e.g. in phase transition problems, capillarity, minimal surfaces [1], [18], [27].

Any absolute or relative minimizer $A$ to $\tilde{\mathcal{G}}$ is known to verify the condition that the sum of the principal curvatures at any point $x \in \partial A \cap \Omega$ equals $g(x)$ (wherever $g$ is continuous) and that the interface $\partial A$ meets the boundary $\partial \Omega$ orthogonally.

Given $\epsilon>0$, the functional $\tilde{\mathcal{G}}$ in (1.1) can be approximated by the more regular relaxed functional [25]

$$
\mathcal{F}_{\epsilon}(v)=\epsilon \int_{\Omega}|\nabla v|^{2} d x+\frac{1}{\epsilon} \int_{\Omega} \Psi(v) d x-\frac{c_{0}}{2} \int_{\Omega} g v d x, \quad \forall v \in H^{1}(\Omega),
$$

in the sense of De Giorgi's $\Gamma$-convergence [13] as $\epsilon \rightarrow 0$. Here, $\Psi(t)=\left(1-t^{2}\right)^{2}$ is a double-well potential which penalizes values of $v$ other than \pm 1 and $c_{0}=$

Received by the editor September 28, 1994 and, in revised form, August 9, 1995.

1991 Mathematics Subject Classification. Primary 35B25, 35J60, 65N30; Secondary 35A35, 49Q05 .

This work was partially supported by MURST (Progetto Nazionale "Equazioni di Evoluzione e Applicazioni Fisico-Matematiche" and "Analisi Numerica e Matematica Computazionale") and CNR (IAN and Contracts 92.00833.01, 93.00564.01) of Italy.

(C)1997 American Mathematical Society 
$\int_{-1}^{1} \sqrt{\Psi(s)} d s$. It follows that any sequence of absolute minimizers $u_{\epsilon}$ of $\mathcal{F}_{\epsilon}$ converges in $L^{1}(\Omega)$, up to a subsequence, to a function $u \in B V(\Omega ;\{-1,1\})$ such that the set $A=\{x \in \Omega: u(x)=1\}$ is an absolute minimizer of $\tilde{\mathcal{G}}$.

The first result of this paper is an essentially $\mathcal{O}\left(\epsilon^{2}\right)$ quasi-optimal interface error estimate for minimizers of $\mathcal{F}_{\epsilon}$ that reads as follows. Let $A \subset \subset \Omega$ be an absolute or relative minimizer of $\tilde{\mathcal{G}}$ with strictly positive principal eigenvalue $\lambda_{1}$ of the second variation of $\tilde{\mathcal{G}}$ ( $A$ is a nondegenerate minimizer). Then there exist a relative minimizer $u_{\epsilon}$ of $\mathcal{F}_{\epsilon}$, a positive constant $C$ depending on $\Omega, g, \lambda_{1}$, and a threshold value $\epsilon_{0}$ (possibly depending also on $A$ and $C$ ) such that, setting $\Sigma_{\epsilon}=\left\{x \in \Omega: u_{\epsilon}(x)=0\right\}$, one has

$$
\operatorname{dist}_{H}\left(\partial A, \Sigma_{\epsilon}\right) \leq C \epsilon^{2}|\log \epsilon|^{2}, \quad \forall \epsilon \leq \epsilon_{0} .
$$

Here, dist $_{H}$ denotes the Hausdorff distance between sets.

The Euler-Lagrange equation associated with $\mathcal{F}_{\epsilon}$ turns out to be a singularly perturbed nonlinear elliptic PDE, which can be solved by finite elements techniques [4], [5]. We then prove that the $\mathcal{O}\left(\epsilon^{2}|\log \epsilon|^{2}\right)$ interface error estimate remains valid also for solutions of these discrete versions of the elliptic PDE, provided the mesh size $h$ decreases with $\epsilon$ at least as $h=\mathcal{O}\left(\epsilon^{5 / 2}\right)$. The discrete problem introduced here actually differs from those presented in [4], [5] in the absence of mass lumping in the potential term. Mass lumping in that term can however be taken into account at the cost of a much stronger requirement on the size of $h$ with respect to $\epsilon$. Results in the same spirit have been recently obtained by Dziuk and Hutchinson [15] for a discrete version of the Plateau problem using a front-tracking approach.

Solutions of the prescribed curvature problem can also be obtained by considering the stationary limit as $t \rightarrow+\infty$ of the evolutionary reaction-diffusion equation associated with the Euler-Lagrange equation,

$$
u_{t}-\Delta u+\frac{1}{2 \epsilon^{2}} \Psi^{\prime}(u)=\frac{c_{0}}{2 \epsilon} g
$$

This so-called Allen-Cahn equation in fact approximates, by its zero level-set, the evolution of a surface moving with normal velocity given by the local mean curvature plus the forcing term $g$ with an error of order $\mathcal{O}\left(\epsilon^{2}|\log \epsilon|^{2}\right)$ [3], [28], [29], [30], [31], [32], [33]. Equation (1.3) has been thoroughly investigated theoretically in the literature; see, e.g., [1], [2], [7], [8], [9], [12], [14], [16], [23] and the references cited therein.

The outline of the paper is as follows. In $\S \S 2$ and 3 we fix some notation and introduce the prescribed curvature problem. The first and second variation of the energy functional are presented in $\S 4$ in order to introduce the concept of nondegeneracy of relative minimizers. The comparison lemma of $\S 5$ is the main tool to establish existence of approximating solutions. Construction of appropriate barriers, based on the formal asymptotics sketched in $\S 6$, is accomplished in $\S 7$. Based on this construction, we obtain the error estimate for the relaxed functional of $\S 8$; the optimality of this estimate is then shown in $\S 9$. A similar error estimate can be obtained for a discrete version of the energy functional, as shown in $\S 10$, following the same conceptual path as for the continuous counterpart. We finish the paper with some remarks and conclusions. 


\section{Notations AND ASSUMPtions}

Let $\Omega \subseteq \mathbf{R}^{n}$ be a bounded open domain with Lipschitz continuous boundary, and let $A \subset \subset \Omega$ with smooth boundary $\Sigma=\partial A$; we require that $A$ lie locally on one side of $\Sigma$ and we suppose for definiteness that $A$ is an open subset of $\Omega$. We require $\Sigma \in \mathcal{C}^{2}$ in order to have continuous principal curvatures $\kappa_{1}, \ldots, \kappa_{n-1}$; additional regularity of $\Sigma$ is implicitly required by the assumption (2.1) below.

The signed distance function with respect to $A$ is defined by

$$
d(x)=\operatorname{dist}\left(x, \mathbf{R}^{n} \backslash A\right)-\operatorname{dist}(x, A)
$$

and satisfies $|d(x)|=\operatorname{dist}(x, \Sigma)$.

Given $D>0$, we define the tubular neighborhood

$$
\mathcal{T}=\{x \in \Omega:|d(x)| \leq D\} .
$$

In view of the $\mathcal{C}^{2}$ regularity of $\Sigma$ and the compact embedding $A \subset \subset \Omega$, we can choose $D$ so small that the signed distance $d$ is smooth over $\mathcal{T}$; it turns out that the projection $s(x)$ of any $x \in \mathcal{T}$ onto $\Sigma$, defined by

$$
\operatorname{dist}(x, s(x))=|d(x)|,
$$

is uniquely determined. Theorems 8.1 and 10.2 will be proved under the following regularity assumptions for $d$ and $g$ :

$$
d \in W^{3, \infty}(\mathcal{T}), \quad g \in W^{3, \infty}(\Omega) .
$$

We recall the definition of the double-well potential $\Psi(t)=\left(1-t^{2}\right)^{2}$, and we set $\psi(t)=\frac{1}{2} \Psi^{\prime}(t)$.

\section{THE PRESCRIBED CURVATURE PROBLEM}

Let us consider a slightly more general form for the energy functional $\tilde{\mathcal{G}}$ obtained by the addition of a boundary term. Namely, let

$$
\tilde{\mathcal{F}}(A)=\mathcal{P}_{\Omega}(A)-\int_{A} g(x) d x+\int_{\partial \Omega \cap \partial A} \mu(x) d \mathcal{H}^{n-1} .
$$

Here, $\mathcal{H}^{n-1}$ denotes the $(n-1)$-dimensional Hausdorff measure and $A$ is any Caccioppoli subset of $\Omega$. Let $\mu \in L^{\infty}(\partial \Omega ;[-1,1])$ be a weight factor for that part of $\partial A$ lying on $\partial \Omega$. It is easy to check that the boundary of minimizers of $\tilde{\mathcal{F}}$ meets the boundary $\partial \Omega$ at an angle given by $\arccos \mu[18]$.

However, in this paper we are only concerned with relative minimizers $A$ compactly contained in $\Omega$, which are clearly not affected by changes in this boundary term. We therefore fix our attention to the particular choice $\mu=-1$ and the corresponding Dirichlet boundary value -1 for $\mathcal{F}_{\epsilon}$. This choice alleviates the boundary layer of minimizers of the relaxed functional, again for compactly contained minimizing sets. Indeed, far outside $A$ such minimizers are espected to assume approximately the value $-1+C \epsilon g$, and we have only an $\mathcal{O}(\epsilon)$ discrepancy with the imposed boundary datum. The constant $C$ depends on the double-well potential, and is actually zero with a nonregular choice of $\Psi$ [33], [29]. Note that the original functional $\tilde{\mathcal{G}}$ corresponds to the choice $\mu=0$.

The functional $\tilde{\mathcal{F}}$ is still relaxed by the functional $\mathcal{F}_{\epsilon}$ defined in (1.2); the only difference lies in the choice of the appropriate Dirichlet boundary condition, which depends on $\mu$ and on the double-well potential [4], [5]. 
We shall restrict attention in the sequel to

$$
\tilde{\mathcal{F}}(A)=\mathcal{H}^{n-1}(\partial A)-\int_{A} g(x) d x .
$$

Correspondingly, we shall impose on $\mathcal{F}_{\epsilon}$ a Dirichlet boundary condition by defining

$$
D\left(\mathcal{F}_{\epsilon}\right)=\left\{v \in H^{1}(\Omega):\left.v\right|_{\partial \Omega}=-1\right\}
$$

and minimizing $\mathcal{F}_{\epsilon}$ over the convex set $D\left(\mathcal{F}_{\epsilon}\right)$. The following $\Gamma$-convergence result is well known [25]. If $\mathcal{F}_{\epsilon}$ is extended to $+\infty$ outside $D\left(\mathcal{F}_{\epsilon}\right)$, then $\mathcal{F}_{\epsilon} \Gamma$-converges to $\frac{c_{0}}{2} \mathcal{F}$ in the $L^{1}(\Omega)$ topology, where $\mathcal{F}$ is defined on $B V(\Omega ;\{-1,1\})$ by

$$
\mathcal{F}(v)=\int_{\Omega}|D v|-\int_{\Omega} g v d x+\int_{\partial \Omega} v d \mathcal{H}^{n-1}
$$

and is extended to $+\infty$ outside $B V(\Omega ;\{-1,1\})$. Here, $\int_{\Omega}|D v|$ stands for the total variation of the $B V$ function $v$ [21]. In terms of relative and absolute minimizers, $\mathcal{F}$ and $\tilde{\mathcal{F}}$ are perfectly interchangeable with the position $A \rightarrow 2 \varphi_{A}-1$, where $\varphi_{A}$ is the usual characteristic function of the set $A$. This $\Gamma$-convergence result implies that any sequence of absolute minimizers of $\mathcal{F}_{\epsilon}$ converge up to a subsequence to an absolute minimizer of $\mathcal{F}$; however, it does not give any information on the rate of convergence.

The relaxed functional $\mathcal{F}_{\epsilon}$ can be easily discretized numerically by using, e.g., conforming piecewise linear finite elements. The resulting discrete functional is shown to $\Gamma$-converge to $\frac{c_{0}}{2} \mathcal{F}[4]$ under the essential condition $h=o(\epsilon)$, where $h$ denotes the mesh size. The sharp error estimate for the continuous relaxed functional $\mathcal{F}_{\epsilon}$ is a crucial step in proving the discrete interface error estimate of $\S 10$.

The Euler-Lagrange equation corresponding to the minimization of $\mathcal{F}_{\epsilon}$ over $D\left(\mathcal{F}_{\epsilon}\right)$ reads as follows:

$$
\mathcal{L}\left(u_{\epsilon}\right)=0 \quad \text { in } \Omega, \quad u_{\epsilon}=-1 \quad \text { on } \partial \Omega,
$$

where

$$
\mathcal{L}(v)=-\epsilon^{2} \Delta v+\psi(v)-\epsilon \frac{c_{0}}{2} g
$$

The operator $\mathcal{L}$ can be regarded as a mapping of $D\left(\mathcal{F}_{\epsilon}\right) \subseteq H^{1}(\Omega)$ into $H^{-1}(\Omega)$.

\section{Stability of SURfaces of PRESCRIBED CURVATURe}

We now turn our attention to the properties satisfied by a relative minimizer $A \subset \subset \Omega$ of $\tilde{\mathcal{F}}$ defined in (3.1). We shall further suppose that the boundary $\Sigma=\partial A$ is of class $C^{2}$, so that the sum of the principal curvatures $\kappa^{m}$ is well defined. Note that this is not a consequence of the regularity of $g$, at least not if the ambient space has dimension $\geq 8$, as the Simons cone [6], [36] example reveals.

We shall consider the first and the second variation $\delta \tilde{\mathcal{F}}_{A}$ and $\delta^{2} \tilde{\mathcal{F}}_{A}$ of $\tilde{\mathcal{F}}$ associated with normal displacements of the boundary $\Sigma$. More precisely, let $n$ denote the outward unit vector normal to $\Sigma$. For any test function $\xi \in C^{2}(\Sigma)$ define

$$
\begin{aligned}
\delta \tilde{\mathcal{F}}_{A}(\xi) & =\frac{d}{d \lambda}\left[\tilde{\mathcal{F}}\left(A_{\lambda \xi}\right)\right]_{\lambda=0}, \\
\delta^{2} \tilde{\mathcal{F}}_{A}(\xi, \xi) & =\frac{d^{2}}{d \lambda^{2}}\left[\tilde{\mathcal{F}}\left(A_{\lambda \xi}\right)\right]_{\lambda=0},
\end{aligned}
$$


where $A_{\lambda \xi}$ is the set whose boundary is obtained by moving any point $x \in \Sigma$ to the new position $x+\lambda \xi(x) n(x)$. The set $A_{\lambda \xi}$ is well defined provided $|\lambda|$ is sufficiently small. A straightforward computation gives the first variation

$$
\delta \tilde{\mathcal{F}}_{A}(\xi)=\int_{\Sigma}\left(\kappa^{m}-g\right) \xi d \mathcal{H}^{n-1},
$$

which provides the usual stationarity condition

$$
\kappa^{m}=g \quad \text { on } \Sigma .
$$

The second variation is given by

$$
\delta^{2} \tilde{\mathcal{F}}_{A}(\xi, \xi)=\int_{\Sigma}\left[\left|\nabla_{\Sigma} \xi\right|^{2}-\left(\nabla g \cdot n+\kappa^{s}\right) \xi^{2}\right] d \mathcal{H}^{n-1},
$$

where $\kappa^{s}$ is the sum of the squares of the principal curvatures, and the notation $\nabla_{\Sigma}$ denotes the tangential gradient of functions defined on $\Sigma$. The symmetric bilinear form on $H^{1}(\Sigma)$ associated with the second variation (4.2) is

$$
\delta^{2} \tilde{\mathcal{F}}_{A}(\xi, \eta)=\left(\nabla_{\Sigma} \xi, \nabla_{\Sigma} \eta\right)_{\Sigma}-\left(\left(\nabla g \cdot n+\kappa^{s}\right) \xi, \eta\right)_{\Sigma},
$$

where $(\cdot, \cdot)_{\Sigma}$ denotes the $L^{2}$ scalar product in $\Sigma$.

The minimality of $A$ implies $\delta^{2} \tilde{\mathcal{F}}_{A}(\xi, \xi) \geq 0$ for any test function $\xi$. The main results of the paper will be proved under a more restrictive nondegeneracy condition.

Definition 4.1. A regular subset $A \subset \subset \Omega$ is a nondegenerate relative minimizer of $\tilde{\mathcal{F}}$ if $(4.1)$ holds and $\delta^{2} \tilde{\mathcal{F}}_{A}(\xi, \xi)>0$ for all $\xi \not \equiv 0$.

Let the linear operator $L: H^{2}(\Sigma) \rightarrow L^{2}(\Sigma)$ be defined by

$$
\delta^{2} \tilde{\mathcal{F}}_{A}(\xi, \eta)=(L \xi, \eta)_{\Sigma}, \quad \forall \eta \in H^{1}(\Sigma),
$$

that is, $L \xi=-\Delta_{\Sigma} \xi-\left(\nabla g \cdot n+\kappa^{s}\right) \xi$, where $\Delta_{\Sigma}$ is the Laplace-Beltrami operator on $\Sigma$ associated with the metric induced by $\mathbf{R}^{n}$.

The eigenvalues of $L$ satisfy $\lambda_{1} \leq \lambda_{2} \leq \cdots$ and $\lambda_{n} \rightarrow+\infty$ as $n \rightarrow \infty$. Let $h \in H^{2}(\Sigma)$ be the eigenfunction associated with the principal eigenvalue $\lambda_{1}$, defined by

$$
-\Delta_{\Sigma} h-\left(\nabla g \cdot n+\kappa^{s}\right) h=\lambda_{1} h
$$

and satisfying $[20, \S 8.12],[11$, p.451]

$$
h_{\text {max }} \geq h(x) \geq h_{\text {min }}>0, \quad \forall x \in \Sigma .
$$

Lemma 4.1. A subset $A \subset \subset \Omega$ satisfying (4.1) is a nondegenerate minimizer if and only if $\lambda_{1}>0$.

Proof. If the test function $\xi$ is normalized so that $\|\xi\|_{L^{2}(\Sigma)}=\|h\|_{L^{2}(\Sigma)}$, then $\delta^{2} \tilde{\mathcal{F}}_{A}(\xi, \xi) \geq \delta^{2} \tilde{\mathcal{F}}_{A}(h, h)=\lambda_{1}(h, h)_{\Sigma}=\lambda_{1}\|h\|_{L^{2}(\Sigma)}^{2}>0$.

Remark 4.1. The eigenfunction $h>0$ satisfying property (4.4) plays an essential role in the construction of the subsolution, since the horizontal shift is modelled accordingly. Instead, the choice of a space-independent shift, as done in [3], [28], [31], [29], leads to a subsolution only if $\nabla g \cdot n+\kappa^{s}<0$ on $\Sigma$. Note that this is a severe restriction which, in turn, implies $\lambda_{1}>0$ in view of (4.2). 


\section{Comparison lemma}

The main tool to establish existence of an approximating solution $u_{\epsilon}$ is the following comparison lemma. It is a variant of known similar results (see [37]).

Lemma 5.1. Suppose that $v_{\epsilon}^{-} \leq v_{\epsilon}^{+} \in W^{2, \infty}(\Omega)$ are sub- and supersolutions to (3.2), that is, satisfy the inequalities $\mathcal{L}\left(v_{\epsilon}^{-}\right) \leq 0$ and $\mathcal{L}\left(v_{\epsilon}^{+}\right) \geq 0$ a.e. in $\Omega$, and also that $v_{\epsilon}^{-} \leq-1 \leq v_{\epsilon}^{+}$on $\partial \Omega$. Then there exists a solution $u_{\epsilon} \in W^{2, \infty}(\Omega)$ of problem (3.2) satisfying $v_{\epsilon}^{-} \leq u_{\epsilon} \leq v_{\epsilon}^{+}$. If, in addition, there exists a positive constant $C$ such that

$$
\mathcal{L}\left(v_{\epsilon}^{-}\right) \leq-C<0 \quad \text { and } \quad \mathcal{L}\left(v_{\epsilon}^{+}\right) \geq C>0,
$$

then $u_{\epsilon}$ can be chosen to be a relative minimizer of the functional $\mathcal{F}_{\epsilon}$.

Proof. Set $K=\left\{v \in H^{1}(\Omega): v_{\epsilon}^{-} \leq v \leq v_{\epsilon}^{+},\left.v\right|_{\partial \Omega}=-1\right\}$. Let $u_{\epsilon} \in K$ be an absolute minimizer of $\mathcal{F}_{\epsilon}$ restricted to $K$ (which is a nonempty closed convex set), which exists by the direct method in Calculus of Variations [24]. Then $u_{\epsilon}$ satisfies the following variational inequality:

$$
\left\langle\mathcal{L} u_{\epsilon}, v-u_{\epsilon}\right\rangle \geq 0 \quad \forall v \in K .
$$

From the regularity theory of elliptic variational inequalities it follows that $u \in$ $W^{2, \infty}(\Omega)$ [19]. Let $x \in \Omega$ be any regular point for $v_{\epsilon}^{-}, v_{\epsilon}^{+}$, and $u_{\epsilon}$. If $v_{\epsilon}^{-}(x)<$ $u_{\epsilon}(x)<v_{\epsilon}^{+}(x)$, we easily get $\mathcal{L}\left(u_{\epsilon}\right)(x)=0$ by appropriately choosing the test function in (5.2). Suppose now $v_{\epsilon}^{-}(x)=u_{\epsilon}(x)<v_{\epsilon}^{+}(x)$. By definition of $\mathcal{L},(3.3)$, we have $\mathcal{L}\left(u_{\epsilon}\right)(x) \leq \mathcal{L}\left(v_{\epsilon}^{-}\right)(x) \leq 0$. On the other hand, we get the opposite inequality $\mathcal{L}\left(u_{\epsilon}\right)(x) \geq 0$ by enforcing (5.2) with an appropriate choice of the test function, and again we obtain $\mathcal{L}\left(u_{\epsilon}\right)(x)=0$. The remaining cases $v_{\epsilon}^{-}(x)<u_{\epsilon}(x)=v_{\epsilon}^{+}(x)$, $v_{\epsilon}^{-}(x)=u_{\epsilon}(x)=v_{\epsilon}^{+}(x)$ can be treated similarly, and we conclude that $u_{\epsilon}$ is a solution of problem (3.2). If we have the stronger conditions (5.1), assumptions $v_{\epsilon}^{-}(x)=u_{\epsilon}(x)$ and $v_{\epsilon}^{+}(x)=u_{\epsilon}(x)$ lead to a contradiction, and we conclude that $u_{\epsilon}$ is contained in the interior of $K$.

\section{Formal ASYMPtOTICS}

A formal asymptotic analysis of the singularly perturbed PDE (3.2) suggests the validity of an optimal quadratic error estimate between the surface of prescribed curvature $\Sigma \subset \subset \Omega$ and the zero level-set of the relaxed solution $u_{\epsilon}$ of (3.2). This error estimate will be proved via the construction of precise barriers suggested by the formal asymptotics mentioned above, which however plays no role in the rigorous proof.

6.1. Shape functions. We preliminarily need to define the shape functions; they appear in the inner expansion, and their asymptotic properties and estimates are crucial for the derivations in $\S 7$. We shall recall them briefly and refer to [3] for details. Let the linear operator $\mathcal{A}: H_{\text {loc }}^{2}(\mathbf{R}) \rightarrow L_{\text {loc }}^{2}(\mathbf{R})$ be defined by

$$
\mathcal{A} \zeta=\zeta^{\prime \prime}-\psi^{\prime}(\gamma) \zeta
$$

where $\gamma$ is the unique strictly increasing solution of $\gamma^{\prime \prime}-\psi(\gamma)=0$ satisfying $\gamma(0)=0$ (for the choice of the potential made in $\S 1$ we actually have $\gamma(y)=\tanh (y)$ ). The 
shape functions are defined by the equations

$$
\begin{aligned}
\mathcal{A} \eta & =\gamma^{\prime}-\frac{c_{0}}{2}, \\
\mathcal{A} \xi^{(1)} & =\eta^{\prime}, \\
\mathcal{A} \xi^{(2)} & =\frac{1}{2} \eta^{2} \psi^{\prime \prime}(\gamma), \\
\mathcal{A} \xi^{(3)} & =y \gamma^{\prime} .
\end{aligned}
$$

Since the right-hand sides (say $f$ ) satisfy the orthogonality condition $\int_{\mathbf{R}} f \gamma^{\prime} d x=0$, any such solution (say $\zeta$ ) actually exists and is unique if we further require $\zeta(0)=0$ and a polynomial growth at infinity [3]. They satisfy the following properties (see $[3, \S 3.2])$ :

$$
\begin{aligned}
\lim _{y \rightarrow \pm \infty} \eta(y) & =\eta_{\infty}=\frac{c_{0}}{2 \psi^{\prime}(1)}, \\
\lim _{y \rightarrow \pm \infty} \xi^{(1)}(y) & =\lim _{y \rightarrow \pm \infty} \xi^{(3)}(y)=0, \\
\lim _{y \rightarrow \pm \infty} \xi^{(2)}(y) & = \pm \xi_{\infty}^{(2)}=\mp \frac{c_{0}^{2} \psi^{\prime \prime}(1)}{8\left(\psi^{\prime}(1)\right)^{3}} .
\end{aligned}
$$

6.2. Inner expansion. We focus our attention here to the sole inner expansion, which furnishes all the important information and allows us to define the building blocks in devising the appropriate shape of the sub- and supersolution.

Let $y=d_{\epsilon} / \epsilon$, where $d_{\epsilon}$ is the signed distance to the set $\Sigma_{\epsilon}=\left\{u_{\epsilon}=0\right\}$, positive inside. Let $\Gamma_{\epsilon}: S \rightarrow \Sigma_{\epsilon}$ be a one-to-one parametrization of $\Sigma_{\epsilon}$, where $S$ is a reference manifold of the same topological type as $\Sigma_{\epsilon}$, assumed to be uniform in $\epsilon$. We also denote by $s: \Omega \rightarrow S$ the projection of any $x \in \Omega$ onto the set $\Sigma_{\epsilon}$, so that

$$
x=\Gamma_{\epsilon}(s(x))-d_{\epsilon}(x) n_{\epsilon}\left(\Gamma_{\epsilon}(s(x))\right),
$$

where $n_{\epsilon}$ denotes the outward unit normal vector to $\Sigma_{\epsilon}$. Setting $U_{\epsilon}(y, s)=u_{\epsilon}(x)$, we assume the existence of asymptotic expansions for $U_{\epsilon}, \Gamma_{\epsilon}, n_{\epsilon}, \kappa_{\epsilon}^{m}, \kappa_{\epsilon}^{s}$ up to the appropriate order in terms of $\epsilon$ :

$$
\begin{gathered}
U_{\epsilon}=U_{0}+\epsilon U_{1}+\epsilon^{2} U_{2}+\mathcal{O}\left(\epsilon^{3}\right), \quad \Gamma_{\epsilon}=\Gamma_{0}+\epsilon \Gamma_{1}+\mathcal{O}\left(\epsilon^{2}\right), \\
n_{\epsilon}=n_{0}+\mathcal{O}(\epsilon), \quad \kappa_{\epsilon}^{m}=\kappa_{0}^{m}+\epsilon \kappa_{1}^{m}+\mathcal{O}\left(\epsilon^{2}\right), \quad \kappa_{\epsilon}^{s}=\kappa_{0}^{s}+\mathcal{O}(\epsilon),
\end{gathered}
$$

and define $\Sigma_{0}=\left\{\Gamma_{0}(s): s \in S\right\}$. By substitution of $U_{\epsilon}$ in (3.2), and collecting all terms of the same order, we obtain equations for each $U_{i}$, which are solvable if and only if an appropriate orthogonality condition for the right-hand sides is satisfied (Fredholm alternative) [3], [29], [33]. In particular, it follows that $\kappa_{0}^{m}=g\left(\Gamma_{0}(s(x))\right)$ and $\kappa_{1}^{m}=0$, which means that the curvature of $\Sigma_{0}$ must equal the forcing term $g$, as expected, so that we are indeed approximating a minimizer of $\tilde{\mathcal{F}}$, and that $\Gamma_{1}=0$, which means that the approximation is actually second-order accurate.

The resulting expressions for $U_{i}, i=0,1,2$, read

$$
\begin{aligned}
& U_{0}=\gamma(y), \\
& U_{1}=g(x) \eta(y), \\
& U_{2}=\left(g^{2}(x)-2 g^{\prime}(s)\right) \xi^{(1)}(y)+g^{2}(x) \xi^{(2)}(y)+\left(\kappa_{0}^{s}(s)-g^{\prime}(s)\right) \xi^{(3)}(y),
\end{aligned}
$$

where for convenience we use $g^{\prime}(s)$ as a shorthand for $-\nabla g\left(\Gamma_{0}(s)\right) \cdot n_{0}\left(\Gamma_{0}(s)\right)$. 
Observe that the function $g$ in the definition of $U_{1}$ is evaluated at the local position $x=\Gamma_{\epsilon}(s)-\epsilon y n_{\epsilon}\left(\Gamma_{\epsilon}(s)\right.$ ) (which actually depends on $\epsilon$ ) rather than on $\Gamma_{0}(s)$. This $\mathcal{O}(\epsilon)$ change affects the subsequent definition of $U_{2}$, but is essential to allow the subsolution constructed according to (6.3) to be extended smoothly far from the interface.

6.3. Boundary layer formal asymptotics. The barrier to be constructed in $\S 7$ has to match the Dirichlet boundary value -1 . For this purpose we need to devise an appropriate shape, which is again suggested by the first three terms of the boundary layer formal asymptotics.

The derivation is quite similar to the case of the inner expansion at the transition layer, but now the Fredholm alternative does not give any orthogonality condition to be satisfied for the right-hand side of the equations defining the shape functions, because the associated homogeneous equation has trivial kernel. It is also possible now to give explicit definitions for the above-mentioned shape functions, which we give in (7.12) of $\S 7.3$, where they are used to actually construct the lower barrier near $\partial \Omega$.

\section{Construction of a subsolution}

Based on the eigenfunction $h$ constructed in $\S 4$, we define for any $\epsilon>0$ on $\mathcal{T}$ the modified signed distance as follows:

$$
d_{\epsilon}^{-}(x)=d(x)-c_{1} \bar{h}(x) \epsilon^{2}|\log \epsilon|^{2},
$$

where $\bar{h}(x)=h(s(x))$ and $c_{1}>0$ is a constant to be chosen later independent of $\epsilon$. We set

$$
\mathcal{T}_{\epsilon}^{-}=\left\{x \in \mathcal{T}:\left|d_{\epsilon}^{-}(x)\right| \leq 2 \delta \epsilon|\log \epsilon|\right\},
$$

where $\delta \geq 3$ and note that $\mathcal{T}_{\epsilon}^{-} \subset \subset \mathcal{T}$ for $\epsilon$ sufficiently small. Since $\bar{h}$ is constant along normal directions, we have $\nabla d \cdot \nabla \bar{h}=0$, hence

$$
\left|\nabla d_{\epsilon}^{-}\right|^{2}=1+c_{1}^{2} \mathcal{O}\left(\epsilon^{4}|\log \epsilon|^{4}\right) \text {. }
$$

Here and throughout this section the notation $f=\mathcal{O}\left(\epsilon^{4}|\log \epsilon|^{4}\right)$ stands for $|f| \leq$ $C \epsilon^{4}|\log \epsilon|^{4}$ for $\epsilon$ sufficiently small, say $\epsilon<\epsilon_{0}$, where $C>0$ is some constant independent of $\epsilon$ and of the constant $c_{1}$, as well as $c_{2}$ and $c_{3}$ to be defined later on. The value $\epsilon_{0}$, instead, might depend on $c_{i}, i=1,2,3$. Note that $c_{i} o(\omega(\epsilon))=$ $\mathcal{O}(\omega(\epsilon)), i=1,2,3$, where $\omega$ is any positive function of $\epsilon$.

Finally, the stretched variable is defined on $\mathcal{T}$ by

$$
y=\frac{d_{\epsilon}^{-}(x)}{\epsilon} .
$$

As in [3], with the important difference of the definition of the modified distance function $d_{\epsilon}^{-}(x)$ and the related stretched variable $y$, the definition of the subsolution $v_{\epsilon}^{-}$is based on the formal asymptotics sketched in $\S 6$.

Also, the existence of a boundary layer for $u_{\epsilon}$, owing to the Dirichlet boundary condition, requires appropriate consideration.

Setting $y_{\epsilon}=\delta|\log \epsilon|$, we need to modify the shape functions $\gamma, \eta, \xi^{(1)}, \xi^{(2)}, \xi^{(3)}$ outside some compact interval $\left[-y_{\epsilon}, y_{\epsilon}\right]$ in order to let them become constant outside the larger interval $\left[-2 y_{\epsilon}, 2 y_{\epsilon}\right]$. This is a necessary step in order to unambiguously 
extend the definition of $v_{\epsilon}^{-}$to the whole $\Omega$. If $\zeta$ is any of the shape functions, denoting $\zeta_{ \pm \infty}=\lim _{y \rightarrow \pm \infty} \zeta(y)$, we define

$$
\zeta_{\epsilon}(y)= \begin{cases}\zeta(y) & \text { if }|y|<y_{\epsilon}, \\ P_{\zeta}^{ \pm}(y) & \text { if } y_{\epsilon} \leq|y| \leq 2 y_{\epsilon}, \\ \zeta_{+\infty} & \text { if } y>2 y_{\epsilon}, \\ \zeta_{-\infty} & \text { if } y<-2 y_{\epsilon},\end{cases}
$$

where $P_{\zeta}^{ \pm}$are two cubic polynomials suitably chosen so as to have $\zeta_{\epsilon} \in \mathcal{C}^{1}(\mathbf{R})$.

We are now ready to define $v_{\epsilon}^{-}$within the tubular neighborhood $\mathcal{T}$ as follows:

$$
v_{\epsilon}^{-}(x)=\gamma_{\epsilon}(y)+\epsilon g(x) \eta_{\epsilon}(y)+\epsilon^{2} \mathbf{b}(x) \cdot \xi_{\epsilon}(y)-c_{2} \epsilon^{3}|\log \epsilon|^{2}, \quad \forall x \in \mathcal{T},
$$

where we used the compact vector notation $\xi_{\epsilon}=\left(\xi_{\epsilon}^{(1)}, \xi_{\epsilon}^{(2)}, \xi_{\epsilon}^{(3)}\right)$ and $\mathbf{b}=\left(g^{2}-2 \nabla d\right.$. $\left.\nabla g, g^{2}, \bar{\kappa}^{s}-\nabla d \cdot \nabla g\right) ; \bar{\kappa}^{s}$ is the extension of $\kappa^{s}$ to $\mathcal{T}$, defined by $\bar{\kappa}^{s}(x)=\kappa^{s}(s(x))$. Here, $c_{2}$ is an appropriate constant to be chosen later.

Since the modified shape functions are constant outside $\mathcal{T}_{\epsilon}^{-}$and in particular $\xi_{ \pm \infty}^{(1)}=\xi_{ \pm \infty}^{(3)}=0$, we can extend $v_{\epsilon}^{-}$on $\Omega \backslash \mathcal{T}_{\epsilon}^{-}$:

$$
v_{\epsilon}^{-}(x)= \begin{cases}1+\epsilon \eta_{\infty} g(x)+\epsilon^{2} \xi_{\infty}^{(2)}(g(x))^{2}-c_{2} \epsilon^{3}|\log \epsilon|^{2} & \text { if } d_{\epsilon}^{-}(x)<-2 \delta \epsilon|\log \epsilon| \\ -1+\epsilon \eta_{\infty} g(x)-\epsilon^{2} \xi_{\infty}^{(2)}(g(x))^{2}-c_{2} \epsilon^{3}|\log \epsilon|^{2} & \text { if } d_{\epsilon}^{-}(x)>2 \delta \epsilon|\log \epsilon|\end{cases}
$$

where we also exploit $\eta_{-\infty}=\eta_{\infty}$ and $\xi_{-\infty}^{(2)}=-\xi_{\infty}^{(2)}$.

The two definitions agree on the common set $\mathcal{T} \backslash \mathcal{T}_{\epsilon}^{-}$.

7.1. Subsolution on $\mathcal{T}_{\epsilon}^{-}$. The most delicate part of this paper is to verify that $v_{\epsilon}^{-}$is actually a subsolution on $\mathcal{T}_{\epsilon}^{-}$with an appropriate choice of the constants $c_{1}, c_{2}$. Note that

$$
d, d_{\epsilon}^{-}=\mathcal{O}(\epsilon|\log \epsilon|) .
$$

We need the following lemma; the proof can be found in [22, Theorem 3.2].

Lemma 7.1. Given any function $w \in C^{2}(\Sigma)$, we can compute the Laplace-Beltrami operator $\Delta_{\Sigma} w$ as follows:

$$
\Delta_{\Sigma} w(s(x))=\Delta(w \circ s)(x), \quad \forall x \in \Sigma,
$$

where we endow $\Sigma$ with the Riemannian metric induced by $\mathbf{R}^{n}$.

For $x \in \mathcal{T}_{\epsilon}^{-}$we apply Lemma 7.1 to the eigenfunction $h$. By Taylor expansion of $\Delta \bar{h}(x)$ about $s(x)$ we get

$$
\Delta \bar{h}(x)=\Delta_{\Sigma} h(s(x))+d \nabla(\Delta \bar{h}(\xi)) \cdot \nabla d=\Delta_{\Sigma} h(s(x))+\mathcal{O}(\epsilon|\log \epsilon|) \quad \forall x \in \mathcal{T}_{\epsilon}^{-},
$$

where the constant in $\mathcal{O}(\epsilon|\log \epsilon|)$ involves three derivatives of $h$ and $\Sigma$.

The following relation holds in $\mathcal{T}_{\epsilon}^{-}[20, \S 14.6]$ :

$$
\Delta d(x)=-\bar{\kappa}^{m}(x)-d(x) \bar{\kappa}^{s}(x)+\mathcal{O}\left(d^{2}(x)\right) .
$$

We can use (7.5) to express the Laplacian of the modified distance as

$$
\begin{aligned}
\Delta d_{\epsilon}^{-} & =\Delta d-c_{1} \Delta_{\Sigma} h(s) \epsilon^{2}|\log \epsilon|^{2}+c_{1} \mathcal{O}\left(d \epsilon^{2}|\log \epsilon|^{2}\right) \\
& =-\bar{\kappa}^{m}-\epsilon y \bar{\kappa}^{s}-c_{1}\left(\bar{h} \bar{\kappa}^{s}+\Delta_{\Sigma} h(s)\right) \epsilon^{2}|\log \epsilon|^{2}+\mathcal{O}\left(\epsilon^{2}|\log \epsilon|^{2}\right) .
\end{aligned}
$$


The derivation, from here on, strictly follows that of [3, $\S 6.3]$. Direct computations yield

$$
\nabla v_{\epsilon}^{-}=\epsilon^{-1} \gamma_{\epsilon}^{\prime} \nabla d_{\epsilon}^{-}+g \eta_{\epsilon}^{\prime} \nabla d_{\epsilon}^{-}+\epsilon \mathbf{b} \cdot \xi_{\epsilon}^{\prime} \nabla d_{\epsilon}^{-}+\epsilon \eta_{\epsilon} \nabla g+\epsilon^{2} \xi_{\epsilon} \cdot \nabla \mathbf{b},
$$

where $\nabla \mathbf{b}$ acts componentwise. Using (7.1) and (2.1) to bound $\nabla \mathbf{b}$ and $\Delta \mathbf{b}$, and noting that $c_{1}^{2} o\left(\epsilon^{\alpha}\right)=\mathcal{O}\left(\epsilon^{\alpha}\right)$, we get

$$
\Delta v_{\epsilon}^{-}=\epsilon^{-2} \gamma_{\epsilon}^{\prime \prime}+\epsilon^{-1} g \eta_{\epsilon}^{\prime \prime}+\epsilon^{-1} \gamma_{\epsilon}^{\prime} \Delta d_{\epsilon}^{-}+g \eta_{\epsilon}^{\prime} \Delta d_{\epsilon}^{-}+\mathbf{b} \cdot \xi_{\epsilon}^{\prime \prime}+2 \eta_{\epsilon}^{\prime} \nabla g \cdot \nabla d_{\epsilon}^{-}+\mathcal{O}(\epsilon) .
$$

Hence, using (7.6), we have

$$
\begin{aligned}
-\epsilon^{2} \Delta v_{\epsilon}^{-}= & -\gamma_{\epsilon}^{\prime \prime}-\epsilon g \eta_{\epsilon}^{\prime \prime}-\epsilon^{2} \mathbf{b} \cdot \xi_{\epsilon}^{\prime \prime}-\left(\epsilon \gamma_{\epsilon}^{\prime}+\epsilon^{2} g \eta_{\epsilon}^{\prime}\right) \Delta d_{\epsilon}^{-}-2 \epsilon^{2} \eta_{\epsilon}^{\prime} \nabla g \cdot \nabla d_{\epsilon}^{-}+\mathcal{O}\left(\epsilon^{3}\right) \\
= & -\gamma_{\epsilon}^{\prime \prime}-\epsilon g \eta_{\epsilon}^{\prime \prime}+\epsilon \gamma_{\epsilon}^{\prime} \bar{\kappa}^{m}-\epsilon^{2} \mathbf{b} \cdot \xi_{\epsilon}^{\prime \prime}-2 \epsilon^{2} \eta_{\epsilon}^{\prime} \nabla g \cdot \nabla d_{\epsilon}^{-}+\epsilon^{2} g \bar{\kappa}^{m} \eta_{\epsilon}^{\prime}+\epsilon^{2} y \gamma_{\epsilon}^{\prime} \bar{\kappa}^{s} \\
& +c_{1} \epsilon^{3}|\log \epsilon|^{2} \gamma_{\epsilon}^{\prime}\left(\bar{h} \bar{\kappa}^{s}+\Delta_{\Sigma} h(s)\right)+\mathcal{O}\left(\epsilon^{3}|\log \epsilon|^{2}\right) .
\end{aligned}
$$

Now observe that, by (4.1) and noting that $\nabla d=\nabla d_{\epsilon}^{-}+c_{1} \mathcal{O}\left(\epsilon^{2}|\log \epsilon|^{2}\right)$, we obtain

$$
\begin{aligned}
g(x) & =g(s(x)+d \nabla d) \\
& =\bar{\kappa}^{m}+d \nabla d \cdot \overline{\nabla g}+\mathcal{O}\left(d^{2}\right) \\
& =\bar{\kappa}^{m}+\epsilon y \nabla d_{\epsilon}^{-} \cdot \overline{\nabla g}+c_{1} \bar{h} \epsilon^{2}|\log \epsilon|^{2} \nabla d_{\epsilon}^{-} \cdot \overline{\nabla g}+\mathcal{O}\left(\epsilon^{2}|\log \epsilon|^{2}\right),
\end{aligned}
$$

where $\overline{\nabla g}(x)=(\nabla g)(s(x))$. Since $y=\mathcal{O}\left(|\log \epsilon|^{2}\right)$, it thus follows that

$$
\epsilon^{2} g \bar{\kappa}^{m} \eta_{\epsilon}^{\prime}=\epsilon^{2} g^{2} \eta_{\epsilon}^{\prime}+\mathcal{O}\left(\epsilon^{3}|\log \epsilon|\right),
$$

and

$$
\epsilon \gamma_{\epsilon}^{\prime} \bar{\kappa}^{m}=\epsilon \gamma_{\epsilon}^{\prime} g-\epsilon^{2} y \gamma_{\epsilon}^{\prime} \nabla d_{\epsilon}^{-} \cdot \overline{\nabla g}-c_{1} \bar{h} \epsilon^{3}|\log \epsilon|^{2} \gamma_{\epsilon}^{\prime} \nabla d_{\epsilon}^{-} \cdot \overline{\nabla g}+\mathcal{O}\left(\epsilon^{3}|\log \epsilon|^{2}\right) .
$$

Inserting (7.7) and (7.8) in the previous expression of $-\epsilon^{2} \Delta v_{\epsilon}^{-}$, we get

$$
\begin{aligned}
-\epsilon^{2} \Delta v_{\epsilon}^{-}= & -\gamma_{\epsilon}^{\prime \prime}-\epsilon g \eta_{\epsilon}^{\prime \prime}+\epsilon \gamma_{\epsilon}^{\prime} g-\epsilon^{2} y \gamma_{\epsilon}^{\prime} \nabla d_{\epsilon}^{-} \cdot \overline{\nabla g}-c_{1} \bar{h} \epsilon^{3}|\log \epsilon|^{2} \gamma_{\epsilon}^{\prime} \nabla d_{\epsilon}^{-} \cdot \overline{\nabla g} \\
& -\epsilon^{2} \mathbf{b} \cdot \xi_{\epsilon}^{\prime \prime}-2 \epsilon^{2} \eta_{\epsilon}^{\prime} \nabla g \cdot \nabla d_{\epsilon}^{-}+\epsilon^{2} g^{2} \eta_{\epsilon}^{\prime}+\epsilon^{2} y \gamma_{\epsilon}^{\prime} \bar{\kappa}^{s} \\
& +c_{1} \epsilon^{3}|\log \epsilon|^{2} \gamma_{\epsilon}^{\prime}\left(\bar{h} \bar{\kappa}^{s}+\Delta_{\Sigma} h(s)\right)+\mathcal{O}\left(\epsilon^{3}|\log \epsilon|^{2}\right) .
\end{aligned}
$$

Using the formula

$$
\begin{aligned}
\psi\left(v_{\epsilon}^{-}\right)= & \psi\left(\gamma_{\epsilon}\right)+\epsilon g \eta_{\epsilon} \psi^{\prime}\left(\gamma_{\epsilon}\right)+\epsilon^{2} \mathbf{b} \cdot \xi_{\epsilon} \psi^{\prime}\left(\gamma_{\epsilon}\right) \\
& +\frac{1}{2} \epsilon^{2} g^{2} \eta_{\epsilon}^{2} \psi^{\prime \prime}\left(\gamma_{\epsilon}\right)-c_{2} \epsilon^{3}|\log \epsilon|^{2} \psi^{\prime}\left(\gamma_{\epsilon}\right)+\mathcal{O}\left(\epsilon^{3}\right),
\end{aligned}
$$

we finally get that

$$
-\epsilon^{2} \Delta v_{\epsilon}^{-}+\psi\left(v_{\epsilon}^{-}\right)-\epsilon \frac{c_{0}}{2} g=\mathrm{I}_{\epsilon}+\epsilon \mathrm{II}_{\epsilon}+\epsilon^{2} \mathrm{III}_{\epsilon}+\epsilon^{3}|\log \epsilon|^{2} \mathrm{IV}_{\epsilon}+\mathcal{O}\left(\epsilon^{3}|\log \epsilon|^{2}\right) \text { in } \mathcal{T}_{\epsilon}^{-}
$$


where, recalling the expression of $\mathbf{b}$ and $\xi_{\epsilon}$,

$$
\begin{aligned}
\mathrm{I}_{\epsilon}= & -\left(\gamma_{\epsilon}^{\prime \prime}-\psi\left(\gamma_{\epsilon}\right)\right), \\
\mathrm{II}_{\epsilon}= & -g\left[\eta_{\epsilon}^{\prime \prime}-\eta_{\epsilon} \psi^{\prime}\left(\gamma_{\epsilon}\right)+\frac{c_{0}}{2}-\gamma_{\epsilon}^{\prime}\right], \\
\mathrm{III}_{\epsilon}= & -\mathbf{b} \cdot \xi_{\epsilon}^{\prime \prime}+\mathbf{b} \cdot \xi_{\epsilon} \psi^{\prime}\left(\gamma_{\epsilon}\right)-2 \eta_{\epsilon}^{\prime} \nabla g \cdot \nabla d+g^{2} \eta_{\epsilon}^{\prime}+y \gamma_{\epsilon}^{\prime} \bar{\kappa}^{s}+\frac{1}{2} g^{2} \eta_{\epsilon}^{2} \psi^{\prime \prime}\left(\gamma_{\epsilon}\right)-y \gamma_{\epsilon}^{\prime} \nabla d \cdot \overline{\nabla g} \\
= & -\left(\bar{\kappa}^{s}-\nabla d \cdot \nabla g\right)\left(\xi_{\epsilon}^{(3) \prime \prime}-\psi^{\prime}\left(\gamma_{\epsilon}\right) \xi_{\epsilon}^{(3)}-y \gamma_{\epsilon}^{\prime}\right)+y \gamma_{\epsilon}^{\prime} \nabla d \cdot(\nabla g-\overline{\nabla g}) \\
& -\left(g^{2}-2 \nabla d \cdot \nabla g\right)\left(\xi_{\epsilon}^{(1) \prime \prime}-\psi^{\prime}\left(\gamma_{\epsilon}\right) \xi_{\epsilon}^{(1)}-\eta_{\epsilon}^{\prime}\right)-g^{2}\left(\xi_{\epsilon}^{(2) \prime \prime}-\psi^{\prime}\left(\gamma_{\epsilon}\right) \xi_{\epsilon}^{(2)}-\frac{1}{2} \eta_{\epsilon}^{2} \psi^{\prime \prime}\left(\gamma_{\epsilon}\right)\right), \\
\mathrm{IV}_{\epsilon}= & c_{1} \gamma_{\epsilon}^{\prime}\left(\bar{h} \bar{\kappa}^{s}+\Delta_{\Sigma} h(s)\right)-c_{2} \psi^{\prime}\left(\gamma_{\epsilon}\right)-c_{1} \bar{h} \gamma_{\epsilon}^{\prime} \nabla d \cdot \overline{\nabla g}=-c_{1} \lambda_{1} \bar{h} \gamma_{\epsilon}^{\prime}-c_{2} \psi^{\prime}\left(\gamma_{\epsilon}\right),
\end{aligned}
$$

where we also use the equality $\nabla d_{\epsilon}^{-}=\nabla d+c_{1} \mathcal{O}\left(\epsilon^{2}|\log \epsilon|^{2}\right)=\nabla d+\mathcal{O}(\epsilon)$ and relation (4.3) for the eigenfunction $h$, with $\nabla d=-n$.

Observe now that $[3]$ in $\mathcal{T}_{\epsilon}^{-}$

$$
\begin{aligned}
& \gamma_{\epsilon}^{\prime \prime}-\psi\left(\gamma_{\epsilon}\right)=o\left(\epsilon^{2 \delta-1}\right), \\
& \eta_{\epsilon}^{\prime \prime}-\psi^{\prime}\left(\gamma_{\epsilon}\right) \eta_{\epsilon}+\frac{c_{0}}{2}-\gamma_{\epsilon}^{\prime}=o\left(\epsilon^{2 \delta-1}\right), \\
& \xi_{\epsilon}^{(3) \prime \prime}-\psi^{\prime}\left(\gamma_{\epsilon}\right) \xi_{\epsilon}^{(3)}-y \gamma_{\epsilon}^{\prime}=\xi_{\epsilon}^{(1) \prime \prime}-\psi^{\prime}\left(\gamma_{\epsilon}\right) \xi_{\epsilon}^{(1)}-\eta_{\epsilon}^{\prime} \\
& \quad=\xi_{\epsilon}^{(2) \prime \prime}-\psi^{\prime}\left(\gamma_{\epsilon}\right) \xi_{\epsilon}^{(2)}-\frac{1}{2} \eta_{\epsilon}^{2} \psi^{\prime \prime}\left(\gamma_{\epsilon}\right)=o\left(\epsilon^{2 \delta-1}\right),
\end{aligned}
$$

for any $y \neq \pm y_{\epsilon}$. Noting that $y \gamma_{\epsilon}^{\prime} \nabla d \cdot(\nabla g-\overline{\nabla g})=\mathcal{O}\left(\epsilon|\log \epsilon|^{2}\right)$, we conclude from the previous estimates that

$$
\mathrm{I}_{\epsilon}+\epsilon \mathrm{II}_{\epsilon}+\epsilon^{2} \mathrm{III}_{\epsilon}=o\left(\epsilon^{2 \delta-1}\right)+\mathcal{O}\left(\epsilon^{3}|\log \epsilon|^{2}\right) \quad \text { in } \mathcal{T}_{\epsilon}^{-} .
$$

Since $\lim _{y \rightarrow \pm \infty} \psi^{\prime}\left(\gamma_{\epsilon}(y)\right)=\psi^{\prime}( \pm 1)=4$, there exists a positive constant $c_{3}$ such that $c_{3} \gamma_{\epsilon}^{\prime}+\psi^{\prime}\left(\gamma_{\epsilon}\right) \geq 2$ on $\mathbf{R}$. By choosing $c_{2}$ large enough and

$$
c_{1}=\frac{c_{3} c_{2}}{\lambda_{1} h_{\min }}>0
$$

we have

$$
\begin{aligned}
\mathcal{L}\left(v_{\epsilon}^{-}\right) & =-\epsilon^{2} \Delta v_{\epsilon}^{-}+\psi\left(v_{\epsilon}^{-}\right)-\epsilon \frac{c_{0}}{2} g=-\epsilon^{3}|\log \epsilon|^{2}\left(c_{1} \lambda_{1} \bar{h} \gamma_{\epsilon}^{\prime}+c_{2} \psi^{\prime}\left(\gamma_{\epsilon}\right)+\mathcal{O}(1)\right) \\
& \leq-\epsilon^{3}|\log \epsilon|^{2}\left(c_{3} c_{2} \gamma_{\epsilon}^{\prime}+c_{2} \psi^{\prime}\left(\gamma_{\epsilon}\right)+\mathcal{O}(1)\right) \leq-C \epsilon^{3}|\log \epsilon|^{2}<0
\end{aligned}
$$

for some constant $C>0$, since the constant in $\mathcal{O}(1)$ does not depend on the constants $c_{1}, c_{2}$. Note that the constant $c_{1}$, which appears in the final error estimate, depends in particular on the nondegeneracy of the minimizer $A$, given by the size of the positive eigenvalue $\lambda_{1}$.

7.2. Subsolution on $\Omega \backslash \mathcal{T}_{\epsilon}^{-}$. Suppose, for definiteness, that $d_{\epsilon}^{-}<-2 \delta \epsilon|\log \epsilon|$. From (2.1) it follows immediately that $-\epsilon^{2} \Delta v_{\epsilon}^{-}=\mathcal{O}\left(\epsilon^{3}\right)$ and, using (6.2), we get

$$
\begin{aligned}
\psi\left(v_{\epsilon}^{-}\right)= & \psi(1)+\epsilon \eta_{\infty} g \psi^{\prime}(1)+\epsilon^{2} \xi_{\infty}^{(2)} g^{2} \psi^{\prime}(1)-c_{2} \epsilon^{3}|\log \epsilon|^{2} \psi^{\prime}(1) \\
& +\frac{1}{2} \epsilon^{2} \eta_{\infty}^{2} g^{2} \psi^{\prime \prime}(1)+\mathcal{O}\left(\epsilon^{3}\right) \\
= & \epsilon \frac{c_{0}}{2} g-c_{2} \epsilon^{3}|\log \epsilon|^{2} \psi^{\prime}(1)+\mathcal{O}\left(\epsilon^{3}\right)
\end{aligned}
$$


Finally,

$$
\begin{aligned}
\mathcal{L}\left(v_{\epsilon}^{-}\right) & =-\epsilon^{2} \Delta v_{\epsilon}^{-}+\psi\left(v_{\epsilon}^{-}\right)-\epsilon \frac{c_{0}}{2} g=-c_{2} \epsilon^{3}|\log \epsilon|^{2} \psi^{\prime}(1)+\mathcal{O}\left(\epsilon^{3}\right) \\
& \leq-C \epsilon^{3}|\log \epsilon|^{2}<0
\end{aligned}
$$

provided we choose $c_{2}$ sufficiently large.

7.3. Comparison with boundary datum. Unfortunately, the constructed subsolution $v_{\epsilon}^{-}$has boundary value given by the second line of (7.3), which is not comparable with the Dirichlet boundary value -1 , for a general $g$. Unless we require $g=0$ on $\partial \Omega$, we are then forced to suitably modify the subsolution $v_{\epsilon}^{-}$in a tubular neighborhood of $\partial \Omega$, and we then need appropriate regularity $\left(W^{3, \infty}\right)$ of $\partial \Omega$.

We shall denote by $\hat{d}$ the (positive) distance function of any point $x \in \Omega$ to the boundary, and by $\hat{y}=\hat{d} \epsilon^{-1}$ the corresponding stretched variable. Let $\hat{\mathcal{T}}_{\epsilon}=\{x \in$ $\Omega: \hat{d}(x) \leq 2 \hat{\delta} \epsilon|\log \epsilon|\}$ for some positive constant $\hat{\delta}$, which is disjoint from $\mathcal{T}_{\epsilon}^{-}$for $\epsilon$ small. The shape of $v_{\epsilon}^{-}$in $\hat{\mathcal{T}}_{\epsilon}$ is again suggested by formal asymptotics (see $\S 6.3$ ). Because of the matching condition with $v_{\epsilon}^{-}$in the interior of $\Omega$ we need to use the first three terms of the formal expansion. More precisely, we define, similarly to $(7.2)$,

$$
v_{\epsilon}^{-}(x)=-1+\epsilon g(x) \hat{\eta}_{\epsilon}(\hat{y})+\epsilon^{2} \hat{\mathbf{b}} \cdot \hat{\xi}_{\epsilon}(\hat{y})-c_{2} \epsilon^{3}|\log \epsilon|^{2}, \quad \forall x \in \hat{\mathcal{T}}_{\epsilon},
$$

where $\hat{\mathbf{b}}=\left(\hat{\kappa}^{m} g-2 \nabla g \cdot \nabla \hat{d}, g^{2}\right), \hat{\xi}_{\epsilon}=\left(\hat{\xi}_{\epsilon}^{(1)}, \hat{\xi}_{\epsilon}^{(2)}\right)$, and $\hat{\kappa}^{m}$ refers to the curvature of $\partial \Omega$. The shape functions $\hat{\eta}_{\epsilon}, \hat{\xi}_{\epsilon}^{(1)}, \hat{\xi}_{\epsilon}^{(2)}$ can be explicitly constructed and are given by

$$
\begin{aligned}
\hat{\eta}_{\epsilon}(\hat{y}) & =\frac{c_{0}}{2 \psi^{\prime}(1)}\left(1-\exp _{\epsilon}(-\sigma \hat{y})\right), \\
\hat{\xi}_{\epsilon}^{(1)}(\hat{y}) & =-\frac{c_{0}}{4 \psi^{\prime}(1)} \hat{y} \exp _{\epsilon}(-\sigma \hat{y}), \\
\hat{\xi}_{\epsilon}^{(2)}(\hat{y}) & =\frac{c_{0}^{2} \psi^{\prime \prime}(1)}{8\left[\psi^{\prime}(1)\right]^{3}}\left[1-\left(\frac{2}{3}+\sigma \hat{y}\right) \exp _{\epsilon}(-\sigma \hat{y})-\frac{1}{3}\left(\exp _{\epsilon}(-\sigma \hat{y})\right)^{2}\right],
\end{aligned}
$$

where $\sigma=\sqrt{\psi^{\prime}(1)}$ and $\exp _{\epsilon}$ denotes the exponential function modified for $\hat{y}>$ $\hat{\delta}|\log \epsilon|$ by a cubic polinomial, as done for the "internal layer" shape functions, so as to vanish for $\hat{y} \geq 2 \hat{\delta}|\log \epsilon|$.

It is clear from this definition that $v_{\epsilon}^{-}(x)<-1$ for any $x \in \partial \Omega$ and that there is consistency with definition (7.3). It only remains to prove that $\mathcal{L} v_{\epsilon}^{-} \leq$ $-C \epsilon^{3}|\log \epsilon|^{2}<0$ in $\hat{\mathcal{T}}_{\epsilon}$. Indeed, if we expand $\mathcal{L} v_{\epsilon}^{-}$as we did in $\mathcal{T}_{\epsilon}^{-}$, we find that the terms of order $\mathcal{O}(1), \mathcal{O}(\epsilon), \mathcal{O}\left(\epsilon^{2}\right)$ essentially vanish, apart for contributions of order $\mathcal{O}\left(\epsilon^{3}\right)$ owing to the truncation of the exponential for $y>\hat{\delta}|\log \epsilon|$; we remain with the $\mathcal{O}\left(\epsilon^{3}|\log \epsilon|^{2}\right)$ term, which has the form $-c_{2} \psi^{\prime}(1) \epsilon^{3}|\log \epsilon|^{2}<0$.

7.4. The approximating solution. The previous arguments can be repeated to construct a supersolution $v_{\epsilon}^{+}$based on the modified distance $d_{\epsilon}^{+}(x)=d(x)+$ $c_{1} \bar{h}(x) \epsilon^{2}|\log \epsilon|^{2}$.

We can now apply the Comparison Lemma 5.1 and deduce that there exists a relative minimum $u_{\epsilon}$ of $\mathcal{F}_{\epsilon}$ satisfying the inequality

$$
v_{\epsilon}^{-} \leq u_{\epsilon} \leq v_{\epsilon}^{+} \quad \text { in } \Omega \text {. }
$$




\section{INTERFACE ERROR ESTIMATE}

Let us denote by dist $_{H}$ the Hausdorff distance between subsets of $\mathbf{R}^{n}$, namely

$$
\operatorname{dist}_{H}(E, F)=\max \left\{\sup _{x \in F} \operatorname{dist}(x, E), \sup _{x \in E} \operatorname{dist}(x, F)\right\} .
$$

Note that $\operatorname{dist}_{H}$ satisfies the following elementary properties:

If $E \subseteq F$ are two different subsets of $\mathbf{R}^{n}$, then

$$
\operatorname{dist}_{H}(E, F)=\sup _{x \in F} \operatorname{dist}(x, E)=\sup _{x \in F \backslash E} \operatorname{dist}(x, E)=\sup _{x \in F \backslash E} \operatorname{dist}(x, \partial E) .
$$

If $A^{-} \subseteq E, F \subseteq A^{+}$, then

$$
\operatorname{dist}_{H}(\partial E, \partial F) \leq \max \left\{\operatorname{dist}_{H}\left(A^{-}, A^{+}\right), \operatorname{dist}_{H}\left(\mathbf{R}^{n} \backslash A^{-}, \mathbf{R}^{n} \backslash A^{+}\right)\right\} .
$$

In fact, if $x \in \partial F \backslash E$, then

$$
\operatorname{dist}(x, \partial E)=\operatorname{dist}(x, E) \leq \operatorname{dist}\left(x, A^{-}\right) \leq \operatorname{dist}_{H}\left(A^{-}, A^{+}\right) .
$$

If $x \in \partial F \cap E$, then

$$
\operatorname{dist}(x, \partial E)=\operatorname{dist}\left(x, \mathbf{R}^{n} \backslash E\right) \leq \operatorname{dist}\left(x, \mathbf{R}^{n} \backslash A^{+}\right) \leq \operatorname{dist}_{H}\left(\mathbf{R}^{n} \backslash A^{-}, \mathbf{R}^{n} \backslash A^{+}\right) .
$$

This reasoning can then be repeated, with the roles of $E$ and $F$ interchanged.

From now on we shall denote by $C$ a positive constant, different from time to time and independent of $\epsilon$.

Theorem 8.1. Assume the regularity assumptions (2.1) and either $\partial \Omega \in W^{3, \infty}$ (i.e., $\hat{d} \in W^{3, \infty}\left(\hat{\mathcal{T}}_{\epsilon}\right)$ ) or $g$ compactly supported in $\Omega$. Given a nondegenerate relative minimizer $A \subset \subset \Omega$ of $\tilde{\mathcal{F}}$, there exist a solution $u_{\epsilon}$ of $(3.2)$, a constant $C$ independent of $\epsilon$ but possibly depending on $\Omega, g, A$ (in particular on $\lambda_{1}$ ), and a constant $\epsilon_{0}>0$ such that

$$
\operatorname{dist}_{H}\left(\Sigma, \Sigma_{\epsilon}\right) \leq C \epsilon^{2}|\log \epsilon|^{2}, \quad \forall \epsilon<\epsilon_{0},
$$

where $\Sigma=\partial A$ and $\Sigma_{\epsilon}=\left\{u_{\epsilon}=0\right\}$.

Proof. Existence of $u_{\epsilon}$ is established in $\S 7.4$. Set $A^{-}=\left\{\gamma\left(\frac{d_{\epsilon}^{-}}{\epsilon}\right)-C_{1} \epsilon \geq 0\right\}, A^{+}=$ $\left\{\gamma\left(\frac{d_{\epsilon}^{+}}{\epsilon}\right)+C_{1} \epsilon \geq 0\right\}, A_{\epsilon}=\left\{u_{\epsilon} \geq 0\right\}$. Choosing $C_{1}$ sufficiently large (independent of $\epsilon$ ), we clearly have $\gamma\left(\frac{d_{\epsilon}^{-}}{\epsilon}\right)-C_{1} \epsilon \leq v_{\epsilon}^{-}$and $\gamma\left(\frac{d_{\epsilon}^{+}}{\epsilon}\right)+C_{1} \epsilon \geq v_{\epsilon}^{+}$. Hence, $A^{-} \subseteq$ $A, A_{\epsilon} \subseteq A^{+}$, and we can use (8.2). Now, for $\epsilon$ sufficiently small, $A^{+} \backslash A^{-} \subseteq \mathcal{T}$, and we can conclude that $\operatorname{dist}_{H}\left(A^{-}, A^{+}\right)$, $\operatorname{dist}_{H}\left(\mathbf{R}^{n} \backslash A^{-}, \mathbf{R}^{n} \backslash A^{+}\right) \leq C \epsilon^{2}|\log \epsilon|^{2}$. To see this, we argue as follows. Fix $x \in A^{+} \backslash A^{-}$. We have $\gamma\left(\frac{d_{\epsilon}^{+}}{\epsilon}\right)+C_{1} \epsilon \geq 0$ and $\gamma\left(\frac{d_{\epsilon}^{-}}{\epsilon}\right)-C_{1} \epsilon<0$, which imply

$$
d_{\epsilon}^{+} \geq-\epsilon \gamma^{-1}\left(C_{1} \epsilon\right) \geq-2 C_{1} \epsilon^{2}, \quad d_{\epsilon}^{-}<2 C_{1} \epsilon^{2} .
$$

Define $y=x+C \epsilon^{2}|\log \epsilon|^{2} \nabla d$ with $C>3 C_{1}+2 c_{1} h_{\max }$. From the properties of the distance function and the definition of $d_{\epsilon}^{-}$and $d_{\epsilon}^{+}$we clearly have $s(x)=s(y)$, and we readily get $d_{\epsilon}^{-}(y)=d_{\epsilon}^{+}(y)-2 c_{1} \bar{h}(x) \epsilon^{2}|\log \epsilon|^{2}>d_{\epsilon}^{+}(x)+3 C_{1} \epsilon^{2}>2 C_{1} \epsilon^{2}$. This implies $\frac{d_{\epsilon}^{-}(y)}{\epsilon}>2 C_{1} \epsilon$, which gives $y \in A^{-}$, since $\gamma\left(2 C_{1} \epsilon\right)>C_{1} \epsilon$ for $\epsilon$ sufficiently small. Since $\operatorname{dist}(x, y)=C \epsilon^{2}|\log \epsilon|^{2}$, we get the result, using (8.1). 


\section{Optimality}

Optimality of the quadratic rate of convergence expressed in Theorem 8.1 can be formally shown by examining the very special case of a circular minimizer, and using formal asymptotics.

Take $n=2$, and select a radially symmetric forcing term $g$ of the special form

$$
g(r)=\alpha r^{2}+\beta r+1-\alpha-\beta,
$$

where $r=|x|$ is the distance from the origin. Since $g(1)=1$, the circle $A=\{r<1\}$ is a stationary point for the functional $\tilde{\mathcal{F}}$ in $(3.1)$. It is not difficult to verify that this circle is the unique absolute minimizer of $\tilde{\mathcal{F}}$ for all $(\alpha, \beta)$ in some neighborhood of $(0,-4)$.

If we express (3.2) for radial symmetry, we obtain for the solution $u_{\epsilon}=u_{\epsilon}(r)$ the equation

$$
\mathrm{I}+\mathrm{II}+\mathrm{III}+\mathrm{IV}:=-\epsilon^{2} r u_{\epsilon}^{\prime \prime}-\epsilon^{2} u_{\epsilon}^{\prime}+r \psi\left(u_{\epsilon}\right)-\frac{c_{0}}{2} \epsilon r g(r)=0 .
$$

We shall denote by $\phi_{\epsilon}$ a root of $u_{\epsilon}(r)$, i.e., $u_{\epsilon}\left(\phi_{\epsilon}\right)=0$, which corresponds to the radius of the approximating circle $\Sigma_{\epsilon}$. Set $\phi_{\epsilon}=1+\sigma_{\epsilon}$.

If we suppose by contradiction that the error between $\Sigma$ and $\Sigma_{\epsilon}$ is more than quadratic in $\epsilon$, we should have

$$
\sigma_{\epsilon}=o\left(\epsilon^{2}\right)
$$

Finally, we introduce the stretched variable $y=\epsilon^{-1}\left(\phi_{\epsilon}-r\right)$, and the stretched solution within the transition interval,

$$
U_{\epsilon}(y)=u_{\epsilon}\left(\phi_{\epsilon}-\epsilon y\right) .
$$

We now intend to develop an accurate formal asymptotic expansion for $U_{\epsilon}$ and consider all the terms of order $\geq \mathcal{O}\left(\epsilon^{3}\right)$. Expand $U_{\epsilon}$ as follows:

$$
U_{\epsilon}=U_{0}+\epsilon U_{1}+\epsilon^{2} U_{2}+\epsilon^{3} U_{3}+o\left(\epsilon^{3}\right) .
$$

Since $U_{\epsilon}(0)=0$, we get the conditions

$$
U_{i}(0)=0, \quad i=0, \ldots, 3 .
$$

We clearly have

$$
\begin{aligned}
r= & 1-\epsilon y+\sigma_{\epsilon}, \\
u_{\epsilon}^{\prime}= & -\frac{1}{\epsilon} U_{\epsilon}^{\prime}, \\
u_{\epsilon}^{\prime \prime}= & \frac{1}{\epsilon^{2}} U_{\epsilon}^{\prime \prime}, \\
g(r)= & 1-\epsilon(2 \alpha+\beta) y+\epsilon^{2} \alpha y^{2}+o\left(\epsilon^{2}\right), \\
\psi\left(U_{\epsilon}\right)= & \psi\left(U_{0}\right)+\left(U_{\epsilon}-U_{0}\right) \psi^{\prime}\left(U_{0}\right)+\frac{1}{2}\left(U_{\epsilon}-U_{0}\right)^{2} \psi^{\prime \prime}\left(U_{0}\right) \\
& +\frac{1}{6}\left(U_{\epsilon}-U_{0}\right)^{3} \psi^{\prime \prime \prime}\left(U_{0}\right)+o\left(\epsilon^{3}\right) \\
= & \psi\left(U_{0}\right)+\epsilon U_{1} \psi^{\prime}\left(U_{0}\right)+\epsilon^{2}\left(U_{2} \psi^{\prime}\left(U_{0}\right)+\frac{1}{2} U_{1}^{2} \psi^{\prime \prime}\left(U_{0}\right)\right) \\
& +\epsilon^{3}\left(U_{3} \psi^{\prime}\left(U_{0}\right)+U_{1} U_{2} \psi^{\prime \prime}\left(U_{0}\right)+\frac{1}{6} U_{1}^{3} \psi^{\prime \prime \prime}\left(U_{0}\right)\right)+o\left(\epsilon^{3}\right) .
\end{aligned}
$$


We can now use these relations to obtain the expansions for each term in (9.1). Using (9.2), (9.4), and (9.6), we get

$$
\begin{aligned}
\mathrm{I} & =-\epsilon^{2}\left(1-\epsilon y+\sigma_{\epsilon}\right) \frac{1}{\epsilon^{2}}\left(U_{0}^{\prime \prime}+\epsilon U_{1}^{\prime \prime}+\epsilon^{2} U_{2}^{\prime \prime}+\epsilon^{3} U_{3}^{\prime \prime}+o\left(\epsilon^{3}\right)\right) \\
& =-U_{0}^{\prime \prime}+\epsilon\left(y U_{0}^{\prime \prime}-U_{1}^{\prime \prime}\right)+\epsilon^{2}\left(y U_{1}^{\prime \prime}-U_{2}^{\prime \prime}\right)-\sigma_{\epsilon} U_{0}^{\prime \prime}+\epsilon^{3}\left(y U_{2}^{\prime \prime}-U_{3}^{\prime \prime}\right)+o\left(\epsilon^{3}\right) .
\end{aligned}
$$

Using (9.2) and (9.5), we get

$$
\mathrm{II}=-\epsilon^{2}\left(-\frac{1}{\epsilon}\left(U_{0}^{\prime}+\epsilon U_{1}^{\prime}+\epsilon^{2} U_{2}^{\prime}+o\left(\epsilon^{2}\right)\right)\right)=\epsilon U_{0}^{\prime}+\epsilon^{2} U_{1}^{\prime}+\epsilon^{3} U_{2}^{\prime}+o\left(\epsilon^{3}\right) .
$$

Using (9.2), (9.4), and (9.8), we get

$$
\begin{aligned}
& \mathrm{III}=\left(1-\epsilon y+\sigma_{\epsilon}\right) \psi\left(U_{\epsilon}\right) \\
& =\psi\left(U_{0}\right)+\epsilon\left(U_{1} \psi^{\prime}\left(U_{0}\right)-y \psi\left(U_{0}\right)\right)+\epsilon^{2}\left(U_{2} \psi^{\prime}\left(U_{0}\right)+\frac{1}{2} U_{1}^{2} \psi^{\prime \prime}\left(U_{0}\right)-y U_{1} \psi^{\prime}\left(U_{0}\right)\right) \\
& +\epsilon^{3}\left(U_{3} \psi^{\prime}\left(U_{0}\right)+U_{1} U_{2} \psi^{\prime \prime}\left(U_{0}\right)+\frac{1}{6} U_{1}^{3} \psi^{\prime \prime \prime}\left(U_{0}\right)-y U_{2} \psi^{\prime}\left(U_{0}\right)-\frac{1}{2} y U_{1}^{2} \psi^{\prime \prime}\left(U_{0}\right)\right) \\
& +o\left(\epsilon^{3}\right)+\sigma_{\epsilon} \psi\left(U_{0}\right) .
\end{aligned}
$$

Using (9.4) and (9.7), we get

$$
\begin{aligned}
\mathrm{IV} & =-\frac{c_{0}}{2} \epsilon\left(1-\epsilon y+\sigma_{\epsilon}\right)\left(1-\epsilon(2 \alpha+\beta) y+\epsilon^{2} \alpha y^{2}+o\left(\epsilon^{2}\right)\right) \\
& =\frac{c_{0}}{2}\left(-\epsilon+\epsilon^{2} y(2 \alpha+\beta+1)-\epsilon^{3} y^{2}(3 \alpha+\beta)\right)+o\left(\epsilon^{3}\right) .
\end{aligned}
$$

We now collect all terms of equal order in (9.9), (9.10), (9.11), (9.12) and equate them to zero.

9.1. Order $\mathcal{O}(1)$. Not surprisingly, the $\mathcal{O}(1)$ terms from (9.9),(9.11), together with condition (9.3), force the choice $U_{0}=\gamma$. This also fortunately cancels out the two terms with $\sigma_{\epsilon}$ in the same two expressions for I and III.

9.2. Order $\mathcal{O}(\epsilon)$. After cancelling two terms, we get the equation for $U_{1}$, recalling (6.1),

$$
\mathcal{A} U_{1}=\gamma^{\prime}-\frac{c_{0}}{2}
$$

whence, as expected, $U_{1}=\eta$.

9.3. Order $\mathcal{O}\left(\epsilon^{2}\right)$. We get

$$
\begin{aligned}
\mathcal{A} U_{2} & =y \mathcal{A} U_{1}+\eta^{\prime}+\frac{1}{2} \eta^{2} \psi^{\prime \prime}(\gamma)+\frac{c_{0}}{2} y(2 \alpha+\beta+1) \\
& =\eta^{\prime}+\frac{1}{2} \eta^{2} \psi^{\prime \prime}(\gamma)+y \gamma^{\prime}+\frac{c_{0}}{2} y(2 \alpha+\beta) .
\end{aligned}
$$

This leads to $U_{2}=\xi^{(1)}+\xi^{(2)}+\xi^{(3)}-(2 \alpha+\beta) \xi^{(4)}$. 
9.4. Order $\mathcal{O}\left(\epsilon^{3}\right)$. We are now only interested in terms involving $\alpha$ and $\beta$. All terms not depending on $\alpha$ or $\beta$ will always be denoted by the generic letter $f$. We obtain, recalling that $\mathcal{A} U_{2}=\frac{c_{0}}{2} y(2 \alpha+\beta)+f$,

$$
\begin{aligned}
\mathcal{A} U_{3} & =\frac{c_{0}}{2} y^{2}(2 \alpha+\beta)-(2 \alpha+\beta)\left(\xi^{(4)}\right)^{\prime}-\eta(2 \alpha+\beta) \xi^{(4)} \psi^{\prime \prime}(\gamma)-\frac{c_{0}}{2} y^{2}(3 \alpha+\beta)+f \\
& =-\frac{c_{0}}{2} \alpha y^{2}-(2 \alpha+\beta)\left(\left(\xi^{(4)}\right)^{\prime}+\eta \xi^{(4)} \psi^{\prime \prime}(\gamma)\right)+f=: f_{\alpha, \beta} .
\end{aligned}
$$

The solvability condition for this self-adjoint equation (see, e.g., [3]) implies

$$
\int_{\mathbf{R}} f_{\alpha, \beta} \gamma^{\prime}=0,
$$

and this has to be true for all $(\alpha, \beta)$ in a neighborhood of $(0,-4)$; hence, by differentiating (9.13) with respect to $\alpha$, keeping $2 \alpha+\beta$ fixed, we get

$$
-\frac{c_{0}}{2} \int_{\mathbf{R}} y^{2} \gamma^{\prime}=0
$$

but this is not possible, since $\gamma^{\prime}>0$, and we get a contradiction. This proves that the error between $\Sigma$ and $\Sigma_{\epsilon}$ cannot in general be better than $\mathcal{O}\left(\epsilon^{2}\right)$, and that our main result is quasi-optimal.

\section{The Discrete PROBlem}

Using conforming piecewise linear finite elements, we shall now introduce a discretized version of problem (3.2)-(3.3) equivalent to a system of nonlinear equations, which can be numerically solved, employing appropriate iterative schemes.

We shall prove, under appropriate restrictions on the mesh size $\left(h^{2}=\mathcal{O}\left(\epsilon^{5}\right)\right)$ a discrete counterpart of Theorem 8.1, e.g., that the boundary $\Sigma$ of the nondegenerate minimizer $A \subset \subset \Omega$ can be approximated with an $\mathcal{O}\left(\epsilon^{2}|\log \epsilon|^{2}\right)$ error by a solution of the discretized problem.

10.1. Notations and assumptions. For the sake of simplicity we shall assume $\Omega$ to be a convex polyhedral domain in $\mathbf{R}^{n}$. Since this implies that $\partial \Omega$ is not regular, we shall require $g$ to be compactly supported in $\Omega$.

For any $h>0$ (meshsize) let $\left\{\mathcal{S}_{h}\right\}_{h}$ be a family of finite element partitions of $\Omega$ into simplices having diameter bounded by $h$ and satisfying the minimum-angle regularity assumption [10]. It is not restrictive to assume $\bar{\Omega}=\bigcup_{T \in \mathcal{S}_{h}} T$. Denote by $\mathcal{N}_{h}=\left\{N_{i}\right\}_{i=1}^{J}$ the set of all vertices of the mesh and suppose that $\mathcal{N}_{h}^{0}=\left\{N_{i}\right\}_{i=1}^{I}$, $I<J$, are the internal vertices.

For the main result of this section we also require the following regularity assumption on $\mathcal{S}_{h}$ :

There exists a constant $C$ independent of $h$ such that for any tetrahedron

(A) $T \in \mathcal{S}_{h}$ the projection of any vertex lies inside the opposite face and has distance bigger than $C h$ from the boundary of the opposite face.

In dimension $n=2$, assumpion (A) can be replaced by the less restrictive requirement:

There exists a constant $C$ independent of $h$ such that for any pair

$\left(\mathrm{A}_{*}\right) \quad$ of adjacent triangles $T_{1}, T_{2} \in \mathcal{S}_{h}$ the sum of the opposite angles does not exceed $\pi-C$. 
Assumption $(\mathrm{A})$ (respectively $\left(\mathrm{A}_{*}\right)$ in dimension $n=2$ ) is a stronger version of the acuteness property of meshes (respectively quasi-acuteness), and implies a discrete version of the comparison principle for the discrete operator without mass lumping (Lemma 10.1).

We shall denote by $V_{h}$ the space of globally continuous piecewise linear functions over $\mathcal{S}_{h}$, and denote $V_{h}^{0}:=V_{h} \cap H_{0}^{1}(\Omega)=\left\{v \in V_{h}:\left.v\right|_{\partial \Omega}=0\right\}$. Let $\phi_{j} \in V_{h}$ denote the usual hat function associated with the node $N_{j}, j=1, \ldots, J$. The functions $\phi_{j}, j=1, \ldots, J$ form the standard canonical basis for the finite element space $V_{h}$. Note that the subset $\left\{\phi_{i}\right\}_{i=1}^{I}$ is a basis for $V_{h}^{0} \subset V_{h}$. Finally, $I_{h}: C^{0}(\bar{\Omega}) \rightarrow V_{h}$ will denote the usual Lagrange interpolation operator, and $\Pi_{h}$ the elliptic projection of any $v \in H^{1}(\Omega)$ onto $V_{h}$ defined by

$$
(\nabla v, \nabla \phi)=\left(\nabla\left(\Pi_{h} v\right), \nabla \phi\right), \quad \forall \phi \in V_{h}^{0}, \quad \Pi_{h} v=I_{h} v \text { on } \partial \Omega .
$$

Denote by $M=\left(m_{i j}\right)$ and by $A=\left(a_{i j}\right)$ the mass and stiffness matrices, defined by

$$
m_{i j}=\int_{\Omega} \phi_{i} \phi_{j} d x, \quad a_{i j}=\int_{\Omega} \nabla \phi_{i} \cdot \nabla \phi_{j} d x, \quad i=1, \ldots, I, \quad j=1, \ldots, J .
$$

It follows by direct computation that for meshes satisfying assumption $(\mathrm{A})$, or $\left(\mathrm{A}_{*}\right)$ if $n=2$, we have

$$
-a_{i j} \geq C h^{-2} m_{i j} \geq 0, \quad i=1, \ldots, I, \quad j=1, \ldots, J, \quad i \neq j,
$$

where $C$ denotes here and below some positive constant independent of $h$ and $\epsilon$, possibly different from line to line.

The discrete version of problem (3.2)-(3.3) can be written in variational form as

$$
\begin{aligned}
& \text { find } v \in K_{h} \text { such that } \\
& \left(\mathcal{L}_{h}(v), \phi\right):=\epsilon^{2}(\nabla v, \nabla \phi)+\left(I_{h} \psi(v), \phi\right)-\epsilon \frac{c_{0}}{2}(g, \phi)_{h}=0, \quad \forall \phi \in V_{h}^{0},
\end{aligned}
$$

where $(\cdot, \cdot)$ denotes the $L^{2}(\Omega)$ scalar product, $(g, \phi)_{h}:=\int_{\Omega} I_{h}(g \phi)$ corresponds to the use of the vertex quadrature rule to approximate the integral, and $K_{h}:=\{v \in$ $\left.V_{h}:\left.v\right|_{\partial \Omega}=-1\right\}$.

10.2. Discrete barriers. We can prove the following lemma.

Lemma 10.1. Suppose that $\mathcal{S}_{h}$ satisfies assumption $(\mathrm{A})$ or $\left(\mathrm{A}_{*}\right)$. Let $v, w \in V_{h}$ with $v \leq w$, and $v\left(N_{i}\right)=w\left(N_{i}\right)$ for some internal node $N_{i} \in \mathcal{N}_{h}^{0}$. Then

$$
\left(\mathcal{L}_{h}(v), \phi_{i}\right) \geq\left(\mathcal{L}_{h}(w), \phi_{i}\right)
$$

if $h=o(\epsilon)$ and $\epsilon$ is sufficiently small.

Proof. Let $v_{j}$ and $w_{j}, j=1, \ldots, J$, denote the nodal values of $v$ and $w$, and $L_{\psi}$ the Lipschitz constant of $\psi$ restricted to $[-2,2]$. The equality $v_{i}=w_{i}$ implies $I_{h}[\psi(v)]\left(N_{i}\right)=I_{h}[\psi(w)]\left(N_{i}\right)$, whence

$$
\begin{aligned}
\left(\mathcal{L}_{h}(w)-\mathcal{L}_{h}(v), \phi_{i}\right) & =\epsilon^{2} \sum_{j \neq i} a_{i j}\left(w_{j}-v_{j}\right)+\sum_{j \neq i} m_{i j}\left(\psi\left(w_{j}\right)-\psi\left(v_{j}\right)\right) \\
& \leq \sum_{j \neq i}\left[\epsilon^{2} a_{i j}\left(w_{j}-v_{j}\right)+m_{i j} L_{\psi}\left(w_{j}-v_{j}\right)\right] \leq 0
\end{aligned}
$$

thanks to property $(10.2)$ and the fact that $h=o(\epsilon)$ ( $j$ ranges from 1 to $J$ in all summations). 
Based on Lemma 10.1, we can now prove the following discrete version of Lemma 5.1 .

Lemma 10.2 (Discrete Comparison Lemma). Assume $\mathcal{S}_{h}$ satisfies assumption (A) or $\left(\mathrm{A}_{*}\right)$. Suppose $v^{-}, v^{+} \in K_{h}$ with $v^{-} \leq v^{+}$, and $\left(\mathcal{L}_{h}\left(v^{-}\right), \phi\right) \leq 0,\left(\mathcal{L}_{h}\left(v^{+}\right), \phi\right) \geq 0$ for all $0 \leq \phi \in V_{h}^{0}$ (i.e., $v^{-}$and $v^{+}$are respectively a discrete subsolution and a discrete supersolution). Then there exists $u \in K_{h}, v^{-} \leq u \leq v^{+}$, a solution of the discrete problem (10.3).

Proof. For this proof, which is based on a fixed point argument, we shall identify any function $v \in K_{h}$ with the vector in $\mathbf{R}^{I}$ of internal nodal values $v_{i}=v\left(N_{i}\right)$, $i=1, \ldots, I$.

Introduce $Q \subset \mathbf{R}^{I}$ to be the closed convex set $Q=\left\{v \in K_{h}: v^{-} \leq v \leq v^{+}\right\}$, define the continuous map $F: \mathbf{R}^{I} \rightarrow \mathbf{R}^{I}$ as

$$
F_{i}(v)=\left(\mathcal{L}_{h}(v), \phi_{i}\right), \quad \forall v \in K_{h}, \quad i=1, \ldots, I,
$$

and finally the map $T: Q \rightarrow Q$ by

$$
T(v)=P_{Q}(v-F(v))
$$

where $P_{Q}: \mathbf{R}^{I} \rightarrow Q$ is the componentwise projection on $Q$.

By the Browder fixed point theorem there exists a fixed point $u \in Q$ for the map $T$. The vector $u$ represents a function (again denoted by $u$ ) in $K_{h}$ satisfying $v^{-} \leq u \leq v^{+}$. It remains to prove that $u$ solves problem (10.3), which is equivalent to showing that $F(u)=0$.

Let $w=u-F(u)$. We claim that $w \in Q$. This would imply the result, since in this case $P_{Q}(w)=w$, which gives $u=T(u)=w$ and hence $F(u)=0$.

To see that $w \in Q$, suppose by contradiction that $w_{i}<v_{i}^{-}$at some node $1 \leq i \leq I$ (the proof is similar if $w_{i}>v_{i}^{+}$). Since $u=P_{Q}(w)$, we have $u_{i}=v_{i}^{-}$, and we can apply Lemma 10.1 to the functions $v^{-} \leq u \in K_{h}$, obtaining

$$
\left(\mathcal{L}_{h}\left(v^{-}\right), \phi_{i}\right) \geq\left(\mathcal{L}_{h}(u), \phi_{i}\right) .
$$

This, in conjunction with the fact that $v^{-}$is a subsolution implies that $F_{i}(u)=$ $\left(\mathcal{L}_{h}(u), \phi_{i}\right) \leq 0$, hence $w_{i}=u_{i}-F_{i}(u) \geq u_{i}=v_{i}^{-}$, which contradicts the assumption on $w_{i}$.

By using the continuous barriers constructed in $\S 7$, by means of the elliptic projection defined in (10.1), we now define the discrete barriers as

$$
v_{\epsilon, h}^{-}=\Pi_{h}\left(v_{\epsilon}^{-}\right), \quad v_{\epsilon, h}^{+}=\Pi_{h}\left(v_{\epsilon}^{+}\right) .
$$

Lemma 10.3 ( $L^{\infty}$ stability and error estimate). Suppose $h^{2}|\log h|=o\left(\epsilon^{2}\right)$; then the discrete barrier $v_{\epsilon, h}^{-}$satisfies the following stability estimates:

$$
\left\|v_{\epsilon, h}^{-}\right\|_{L^{\infty}(\Omega)} \leq 2, \quad\left\|\nabla v_{\epsilon, h}^{-}\right\|_{L^{\infty}(\Omega)} \leq \frac{C}{\epsilon},
$$

for sufficiently small $\epsilon$ and $h$. Moreover, the following $L^{\infty}(\Omega)$ error estimate holds:

$$
\left\|v_{\epsilon}^{-}-v_{\epsilon, h}^{-}\right\|_{L^{\infty}(\Omega)} \leq C h^{2}|\log h|\left\|D^{2} v_{\epsilon}^{-}\right\|_{L^{\infty}(\Omega)} \leq C \frac{h^{2}}{\epsilon^{2}}|\log h| .
$$

Similar estimates are valid for $v_{\epsilon, h}^{+}$. 
Proof. The first inequality in (10.6) has been proved by Nitsche [26] and Scott [35] for the elliptic projection; $\left\|D^{2} v_{\epsilon}^{-}\right\|_{L^{\infty}(\Omega)} \leq C \epsilon^{-2}$ follows from the construction of $v_{\epsilon}^{-}$. We also have the following $W^{1, \infty}$ stability estimate (Rannacher, Scott [34]):

$$
\left\|\nabla v_{\epsilon, h}^{-}\right\|_{L^{\infty}(\Omega)} \leq C\left\|\nabla v_{\epsilon}^{-}\right\|_{L^{\infty}(\Omega)},
$$

which immediately gives the second stability estimate in (10.5) in view of the construction of the continuous barrier $v_{\epsilon}^{-}$. Finally, the first estimate in (10.5) follows from (10.6), the hypothesis on $h$, and the $L^{\infty}$ bound $\left\|v_{\epsilon}^{-}\right\|_{L^{\infty}(\Omega)} \leq \frac{3}{2}$ clearly valid for $\epsilon$ small enough.

Using Lemma 10.3, we are in a position to prove that $v_{\epsilon, h}^{-}$is a discrete subsolution.

Theorem 10.1. Under the following restriction on $h$,

$$
h=\mathcal{O}\left(\epsilon^{5 / 2}\right),
$$

the discrete function $v_{\epsilon, h}^{-}$is a discrete subsolution, i.e., $\left(\mathcal{L}_{h}\left(v_{\epsilon, h}^{-}\right), \phi\right) \leq 0$ for all $0 \leq \phi \in V_{h}^{0}$.

Proof. We decompose $\left(\mathcal{L}_{h}\left(v_{\epsilon, h}^{-}\right), \phi\right)$ as follows:

$$
\begin{aligned}
\left(\mathcal{L}_{h}\left(v_{\epsilon, h}^{-}\right), \phi\right)= & \left(\mathcal{L}\left(v_{\epsilon}^{-}\right), \phi\right)+\left[\left(I_{h} \psi\left(v_{\epsilon, h}^{-}\right), \phi\right)-\left(\psi\left(v_{\epsilon, h}^{-}\right), \phi\right)\right] \\
& +\left[\left(\psi\left(v_{\epsilon, h}^{-}\right), \phi\right)-\left(\psi\left(v_{\epsilon}^{-}\right), \phi\right)\right] \\
& +\epsilon \frac{c_{0}}{2}\left[(g, \phi)-(g, \phi)_{h}\right]=: \mathrm{I}+\mathrm{II}+\mathrm{III}+\mathrm{IV} .
\end{aligned}
$$

From $\S \S 7.1,7.2,7.3$, we have $I \leq-C \epsilon^{3}|\log \epsilon|^{2}\|\phi\|_{L^{1}(\Omega)}$. Let $L_{\psi^{\prime}}$ be the Lipschitz constant of $\psi^{\prime}$ restricted to $[-2,2]$. Using standard properties of the interpolation operator $I_{h}$, in conjunction with (10.5), we can estimate

$$
\begin{aligned}
|\mathrm{II}| & \leq\left\|I_{h} \psi\left(v_{\epsilon, h}^{-}\right)-\psi\left(v_{\epsilon, h}^{-}\right)\right\|_{L^{\infty}(\Omega)}\|\phi\|_{L^{1}(\Omega)} \leq C h^{2} \max _{T \in \mathcal{S}_{h}}\left\|D^{2} \psi\left(v_{\epsilon, h}^{-}\right)\right\|_{L^{\infty}(T)}\|\phi\|_{L^{1}(\Omega)} \\
& \leq C h^{2} L_{\psi^{\prime}}\left\|\nabla v_{\epsilon, h}^{-}\right\|_{L^{\infty}(\Omega)}^{2}\|\phi\|_{L^{1}(\Omega)} \leq C \frac{h^{2}}{\epsilon^{2}}\|\phi\|_{L^{1}(\Omega)} .
\end{aligned}
$$

Using (10.6), we have

$$
\begin{aligned}
|\mathrm{III}| & \leq\left\|\psi\left(v_{\epsilon, h}^{-}\right)-\psi\left(v_{\epsilon}^{-}\right)\right\|_{L^{\infty}(\Omega)}\|\phi\|_{L^{1}(\Omega)} \leq L_{\psi}\left\|v_{\epsilon, h}^{-}-v_{\epsilon}^{-}\right\|_{L^{\infty}(\Omega)}\|\phi\|_{L^{1}(\Omega)} \\
& \leq C \frac{h^{2}}{\epsilon^{2}}|\log h|\|\phi\|_{L^{1}(\Omega)} .
\end{aligned}
$$

Finally, in view of the regularity of $g$ and the inverse inequality $\|\nabla \phi\|_{L^{1}(T)} \leq$ $\frac{C}{h_{T}}\|\phi\|_{L^{1}(T)}$, for any $T \in \mathcal{S}_{h}$, where $h_{T}$ is the diameter of $T$, the quadrature error can be bounded as follows:

$$
\begin{aligned}
|\mathrm{IV}| & \leq C \epsilon \sum_{T \in \mathcal{S}_{h}} h_{T}^{2}\left\|D^{2}(g \phi)\right\|_{L^{1}(T)} \\
& \leq C \epsilon \sum_{T \in \mathcal{S}_{h}} h_{T}^{2}\left[\|\nabla g\|_{L^{\infty}(\Omega)}\|\nabla \phi\|_{L^{1}(T)}+\left\|D^{2} g\right\|_{L^{\infty}(\Omega)}\|\phi\|_{L^{1}(T)}\right] \\
& \leq C \epsilon h\|\phi\|_{L^{1}(\Omega)} .
\end{aligned}
$$


The terms II and III can be controlled by term I if we require

$$
\frac{h^{2}}{\epsilon^{2}}|\log h|=\mathcal{O}\left(\epsilon^{3}|\log \epsilon|^{2}\right),
$$

which is seen true by using (10.7). Term I also controls IV, as can be seen by exploiting (10.7).

10.3. The discrete solution. The discrete barriers $v_{\epsilon, h}^{-}, v_{\epsilon, h}^{+}$defined in (10.4) cannot be directly employed in Lemma 10.2, since they do not belong to the affine space $K_{h}$. We can however simply modify, e.g., $v_{\epsilon, h}^{-}$by raising the value on the boundary nodes to be -1 . After this modification $v_{\epsilon, h}^{-}$is a fortiori a subsolution (apply Lemma 10.1 to the original and the modified $v_{\epsilon, h}^{-}$).

The requirement $v_{\epsilon, h}^{-} \leq v_{\epsilon, h}^{+}$is a consequence of the fact that $v_{\epsilon}^{+}-v_{\epsilon}^{-}>$ $C \epsilon^{3}|\log \epsilon|^{2}$ (direct verification) by using the error estimate (10.6) and the relation (10.7).

We can finally apply Lemma 10.2 to get a solution $v_{\epsilon, h}^{-} \leq u_{\epsilon, h} \leq v_{\epsilon, h}^{+}$of problem $(10.3)$.

10.4. Discrete interface error estimate. The constructed discrete barriers $v_{\epsilon, h}^{-}$ and $v_{\epsilon, h}^{+}$allow us to prove a discrete equivalent of Theorem 8.1 for the Hausdorff distance between the exact prescribed curvature surface $\Sigma$ and the discrete surface defined by $\Sigma_{\epsilon, h}:=\left\{u_{\epsilon, h}=0\right\}$.

Theorem 10.2. Under the hypotheses of Theorem 8.1, with $g$ compactly supported in $\Omega$, and under the assumption $(\mathrm{A})$ or $\left(\mathrm{A}_{*}\right)$ for the mesh $\mathcal{S}_{h}$ and the relation (10.7) between the relaxation parameter $\epsilon$ and the mesh size $h$, there exist a constant $C$ independent of $\epsilon$ and $\epsilon_{0}>0$ such that

$$
\operatorname{dist}_{H}\left(\Sigma, \Sigma_{\epsilon, h}\right) \leq C \epsilon^{2}|\log \epsilon|^{2}, \quad \forall \epsilon<\epsilon_{0}
$$

Proof. Sticking with the notation of Theorem 8.1, we claim that $A^{-} \subseteq\left\{v_{\epsilon, h}^{-} \geq 0\right\}$ $\subset\left\{v_{\epsilon, h}^{+} \geq 0\right\} \subseteq A^{+}$. This will immediately imply the result, thanks to property (8.2) of the Hausdorff distance and the relation $v_{\epsilon, h}^{-} \leq u_{\epsilon, h} \leq v_{\epsilon, h}^{+}$.

To prove the claim, simply observe that

$$
\begin{aligned}
v_{\epsilon, h}^{-}(x) & \geq v_{\epsilon}^{-}(x)-\left\|v_{\epsilon, h}^{-}-v_{\epsilon}^{-}\right\|_{L^{\infty}(\Omega)} \\
& \geq \gamma_{\epsilon}\left(\frac{d_{\epsilon}^{-}(x)}{\epsilon}\right)-2 \epsilon\|g\|_{L^{\infty}(\Omega)}\|\eta\|_{L^{\infty}(\Omega)}-C \frac{h^{2}}{\epsilon^{2}}|\log h| \\
& \geq \gamma_{\epsilon}\left(\frac{d_{\epsilon}^{-}(x)}{\epsilon}\right)-C \epsilon,
\end{aligned}
$$

where we used definition (7.2) of $v_{\epsilon}^{-}$, estimate (10.6) and assumption (10.7). In view of the definition of $A^{-}$with $C_{1}$ large enough we get the first inclusion. The second one follows similarly.

\section{A NUMERICAL SIMULATION}

For the sake of completeness we present a numerical simulation included in [5] for the solution of a prescribed curvature problem.

Set $\Omega=(-4,4) \times(-4,4)$ and take $g(x)=-|x|+5 / 2 ;$ then $A=\{x \in \Omega:|x|<2\}$ is a nondegenerate absolute minimizer of $\tilde{\mathcal{F}}$ as defined in (3.1). 


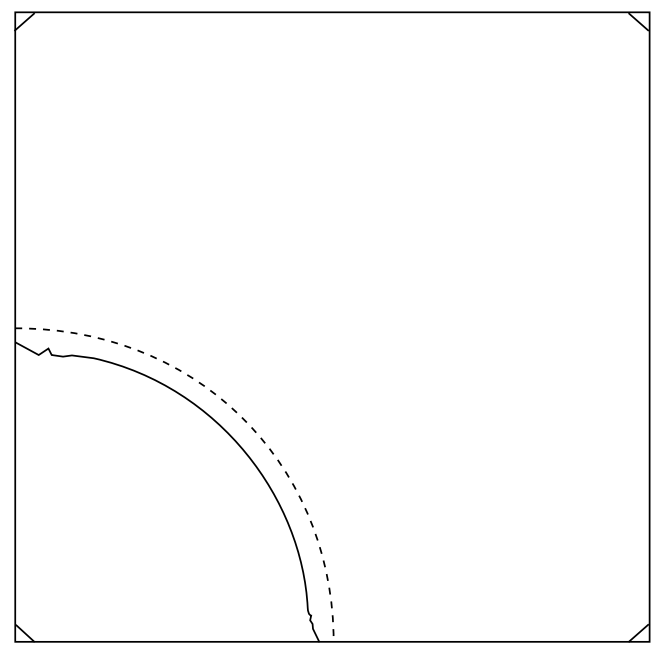

FiguRE 11.1. Exact solution (dashed line) and discrete solution (solid line)

We discretize $\Omega$ with a uniform unstructured triangular mesh of size $h=0.1$, fix $\epsilon=0.17$ and solve the discrete problem (10.3) with the addition of a mass lumping on the nonlinear term involving $\psi$ (see Conclusions, $\S 12$ ) by using a nonlinear Jacobi iteration scheme. We enforce the symmetry of the problem by solving (10.3) on the quadrant $(0,4) \times(0,4)$ with homogeneous Neumann boundary conditions on the coordinate axes. The exact solution $\partial A$ and the computed solution $\Sigma_{\epsilon, h}$ are shown in Figure 11.1.

\section{Conclusions}

The boundary $\partial \Omega$ and the choice of the boundary condition for problem (3.2) plays an important role in Theorem 8.1. Other different boundary conditions such as homogeneous Neumann or general Dirichlet conditions could however be considered with only slight changes in the proofs, if we keep requiring the relative compactness of the minimizer $A \subset \subset \Omega$.

It seems not at all trivial to relax this compactness assumption, unless specific choices for the boundary condition (Homogeneous Neumann) and on $\partial \Omega$ (e.g. planar where $\Sigma$ intersects $\partial \Omega$ ) are taken. The most interesting case of a general Dirichlet boundary condition, which leads to prescribed contact angle for the minimizer to $\partial \Omega$, seems to require a precise construction of the subsolution in the neighborhood of the contact set $\Sigma \cap \partial \Omega$. Moreover, the definition of nondegenerate minimizer has to be reformulated, since now the manifold $\Sigma \cap \Omega$ has a boundary.

The discrete problem formulated in (10.3) does not include mass lumping for the reaction term $\psi(v)$, as was done in [4], [5], leading to a nondiagonal mass matrix in the resulting nonlinear system. Indeed, the use of mass lumping can only be taken into account by a stricter restriction $h=\mathcal{O}\left(\epsilon^{4}\right)$, since the difference $V:=\left(I_{h} \psi\left(v_{\epsilon, h}^{-}\right), \phi\right)_{h}-\left(I_{h} \psi\left(v_{\epsilon, h}^{-}\right), \phi\right)$ now arising in the proof of Theorem $10.1 \mathrm{can}$ 
be bounded by

$$
|V| \leq C \sum_{T \in \mathcal{S}_{h}} h_{T}^{2}\left\|\nabla \psi\left(v_{\epsilon, h}^{-}\right)\right\|_{L^{\infty}(\Omega)}\|\nabla \phi\|_{L^{1}(T)} \leq C \frac{h}{\epsilon}\|\phi\|_{L^{1}(\Omega)}
$$

and can be controlled by term I if $h=\mathcal{O}\left(\epsilon^{4}\right)$.

\section{REFERENCES}

1. S.M. Allen and J.W. Cahn, A macroscopic theory for antiphase boundary motion and its application to antiphase domain coarsing, Acta Metall. 27 (1979), 1085-1095

2. G. Barles, H.-M. Soner, and P.E. Souganidis, Front propagation and phase field theory, SIAM J. Control Optim. 31 (1993), 439-469. MR 94c:35005

3. G. Bellettini and M. Paolini, Quasi-optimal error estimates for the mean curvature flow with a forcing term, Differential Integral Equations 8 (1995), 735-752. MR 95h:35021

4. G. Bellettini, M. Paolini, and C. Verdi, $\Gamma$-convergence of discrete approximations to interfaces with prescribed mean curvature, Atti Accad. Naz. Lincei Cl. Sci. Fis. Mat. Natur. Rend. Lincei (9) Mat. Appl. 1 (1990), 317-328. MR 92a:49019

5. Numerical minimization of geometrical type problems related to calculus of variations, Calcolo 27 (1990), 251-278. MR 92i:65103

6. E. Bombieri, E. De Giorgi, and E. Giusti, Minimal cones and the Bernstein problem, Invent. Math. 7 (1969), 243-268. MR 40:3445

7. L. Bronsard and R.V. Kohn, Motion by mean curvature as the singular limit of GinzburgLandau dynamics, J. Differential Equations 90 (1991), 211-237. MR 92d:35037

8. X. Chen, Generation and propagation of interfaces in reaction-diffusion equations, J. Differential Equations 96 (1992), 116-141. MR 92m:35129

9. X. Chen and C.M. Elliott, Asymptotics for a parabolic double obstacle problem, Proc. Roy. Soc. London Ser. A 444 (1994), 429-445. MR 95f:35128

10. P.G. Ciarlet, The Finite Element Method for Elliptic Problems, North-Holland, Amsterdam, 1978. MR 58:25001

11. R. Courant and D. Hilbert, Methods of Mathematical Physics, Volume I, Interscience Publishers Inc., New York, 1953. MR 16:426a

12. E. De Giorgi, Congetture sui limiti delle soluzioni di alcune equazioni paraboliche quasi lineari, Nonlinear Analysis. A Tribute in Honour of G. Prodi, S.N.S. Quaderni, Pisa, 1991, pp. 173187.

13. E. De Giorgi and T. Franzoni, Su un tipo di convergenza variazionale, Atti Accad. Naz. Lincei Rend. Cl. Sci. Fis. Mat. Natur. (8) 58 (1975), 842-850. MR 56:6503

14. P. De Mottoni and M. Schatzman, Geometrical evolution of developed interfaces, Trans. Amer. Math. Soc. 347 (1995), 1533-1589

15. G. Dziuk and J.E. Hutchinson, On the approximation of unstable parametric minimal surfaces, Calc. Var. Partial Differential Equations 4 (1996), 27-58. CMP 96:09

16. L.C. Evans, H.-M. Soner, and P.E. Souganidis, Phase transitions and generalized motion by mean curvature, Comm. Pure Appl. Math. 45 (1992), 1097-1123. MR 93g:35064

17. H. Federer, Geometric Measure Theory, Springer-Verlag, Berlin, 1968. MR 41:1976

18. R. Finn, Equilibrium Capillary Surfaces, Springer-Verlag, Berlin, 1986. MR 88f:49001

19. A. Friedman, Variational Principles and Free Boundary Problems, Wiley, New York, 1982. MR 84e:35153

20. D. Gilbarg and N.S. Trudinger, Elliptic Partial Differential Equations of Second Order, Springer-Verlag, Berlin, 1983. MR 86c:35035

21. E. Giusti, Minimal Surfaces and Functions of Bounded Variation, Birkhäuser, Boston, 1984. MR 87a:58041

22. S. Helgason, Groups and Geometric Analysis, Academic Press, Orlando, Florida, 1984. MR 86c: 22017

23. T. Ilmanen, Convergence of the Allen-Cahn equation to Brakke's motion by mean curvature, J. Differential Geom. 38 (1993), 417-461. MR 94h:58051

24. H. Lewy, Aspects of the Calculus of Variations, University of California Press, Berkeley, 1939

25. L. Modica and S. Mortola, Un esempio di $\Gamma$-convergenza, Boll. Un. Mat. Ital. B (5) 14 (1977), 285-299. MR 56:3704 
26. J. Nitsche, $L_{\infty}$-convergence of finite element approximations, Mathematical Aspects of Finite Element Methods, Lecture Notes in Math., 606., Springer-Verlag, Berlin, 1977, pp. 261-274. MR 58:8351

27. J.C.C. Nitsche, Lectures on Minimal Surfaces, Volume 1, Cambridge University Press, Cambridge, 1989. MR 90m:49031

28. R.H. Nochetto, M. Paolini, and C. Verdi, Sharp error analysis for curvature dependent evolving fronts, Math. Models Methods Appl. Sci. 3 (1993), 711-723. MR 94k:35028

29. - Optimal interface error estimates for the mean curvature flow, Ann. Scuola Norm. Sup. Pisa Cl. Sci. (4) 21 (1994), 193-212. MR 96b:35020

30. _ Quadratic rate of convergence for curvature dependent smooth interfaces: a simple proof, Appl. Math. Lett. 7 (1994), 59-63. MR 96d:35067

31. __ Double obstacle formulation with variable relaxation parameter for smooth geometric front evolutions: asymptotic interface error estimates, Asymptotic Anal. 10 (1995), 173-198. MR 96b:35238

32. - A dynamic mesh algorithm for curvature dependent evolving interfaces, J. Comput. Phys. 123 (1996), 296-310. CMP 96:07

33. M. Paolini and C. Verdi, Asymptotic and numerical analyses of the mean curvature flow with a space-dependent relaxation parameter, Asymptotic Anal. 5 (1992), 553-574. MR 93h:35101

34. R. Rannacher and R. Scott, Some optimal error estimates for piecewise linear finite element approximations, Math. Comp. 38 (1982), 437-445. MR 83e:65180

35. R. Scott, Optimal $L^{\infty}$ estimates for the finite element method on irregular meshes, Math. Comp. 32 (1978), 681-697. MR 55:9560

36. J. Simons, Minimal varieties in Riemannian manifolds, Ann. of Math. (2) 88 (1968), 62-105. MR 38:1617

37. J. Smoller, Shock Waves and Reaction-Diffusion Equations, Springer-Verlag, New York, 1983. MR 84d:35002

Dipartimento di Matematica e Informatica, Università di Udine, 33100, Udine, Italy E-mail address: paolini@dimi.uniud.it 